\title{
Investigation on rod-airfoil noise with high-order cell-centered finite difference method and acoustic analogy
}

\author{
Yao Jin ${ }^{\mathrm{a}}$, Fei Liao ${ }^{\mathrm{a}, \mathrm{b}, *}$, Jinsheng Cai ${ }^{\mathrm{a}}$, Philip J. Morris ${ }^{\mathrm{c}}$ \\ a School of Aeronautics, Northwestern Polytechnical University, Xi'an, 710072, China \\ b State Key Laboratory of Nonlinear Mechanics, Institute of Mechanics, Chinese Academy of Sciences, Beijing, 100190, China \\ ${ }^{\mathrm{c}}$ Department of Aerospace Engineering, Pennsylvania State University, University Park, PA, 16802, USA
}

\section{A R T I C L E I N F O}

\section{Article history:}

Received 27 July 2019

Received in revised form 20 March 2020

Accepted 15 April 2020

Available online 5 May 2020

Communicated by Damiano Casalino

\section{Keywords:}

Aeroacoustics

Rod-airfoil configuration

Cell-centered finite difference method

Delayed detached eddy simulation

FW-H acoustic analogy

\begin{abstract}
A B S T R A C T
This paper focuses on the radiation noise of a rod-airfoil configuration based on a high-order cellcentered finite difference method (CCFDM) and Ffowcs Williams-Hawkings (FW-H) acoustic analogy. To reduce numerical dissipation and dispersion, a class of optimized compact schemes is firstly proposed and then validated with benchmark cases from computational aeroacoustic workshops. For turbulence, the delayed detached eddy simulation (DDES) with Spalart-Allmaras model is adopted and is validated through a canonical periodic hill problem. For the far-field radiated acoustics, the FW-H acoustic analogy is utilized with validation of laminar flow past NACA0012. Finally, a rod-airfoil configuration is investigated. The results show reasonable agreement with the experimental data in terms of both nearfield aerodynamics and far-field acoustics. The interaction between the upstream turbulence wake from the rod and the downstream airfoil is evidenced to be the dominant contributor to the far-field noise.

(c) 2020 Elsevier Masson SAS. All rights reserved.
\end{abstract}

\section{Introduction}

Computational aeroacoustics (CAA) is a topic of considerable interest to the computational fluid dynamics (CFD) community. The goals in CAA mainly involve three aspects: developing computational approaches [1] for noise propagation and radiation; investigating the noise generation mechanism of practical configurations in industry [2] and exploring noise-control concepts and strategies [3]. Among all, the first goal lays the foundation and is the main focus in this paper.

One concern for CAA tools is the computational efficiency and this motivates us to focus on the finite difference method (FDM) on structured grid where the high-order accuracy can be realized dimensionally by dimensionally. This owns to the coordinate transformation between Cartesian and curvilinear coordinates. But, a constraint is invoked in this process, which is named geometric conservation law (GCL) [4]. Particular attention should be paid to the satisfaction of GCL condition in order to achieve high-order accuracy. Otherwise, the grid induced error will contaminate the flow-field. It is reported [5][6] that a cell-centered finite difference method (CCFDM) and its companion cell-centered symmetric conservative metrics method (CCSCMM) can retain high accuracy and non-violation of GCL. This method will be further developed for aeroacoustics.

The first consideration is to find appropriate spatial schemes for aeroacoustics. The acoustic fluctuations are usually small in the magnitude and can propagate for long distances with nearly no damping. Yet, in conventional CFD tools, the excessive truncation error resulting from the low-order numerical methods will overwhelm the acoustic signals. This motivates the development of low-dissipation and low-dispersion schemes, such as compact schemes [7] and dispersion-relation-preservation scheme (DRP) [8]. In the spirit of DRP scheme, the compact schemes [7] can be further optimized to achieve higher spectral properties [9]. But, these schemes [7][8][9] cannot be used directly because they are in the form of derivatives whereas interpolations are demanding in the framework of CCFDM [5][6]. A class of dissipative compact interpolations [10] is given where one free parameter provides controllable dissipation. In the present paper, new attempt is given on optimization of the compact interpolations so as to achieve more superior spectral properties.

The second consideration is associated with unsteady separated turbulence flows. The direct numerical simulation (DNS) capturing all the physically dynamic scales and large eddy simulation (LES) mainly capturing the energy-containing motions are accurate and reliable.

\footnotetext{
* Corresponding author at: School of Aeronautics, Northwestern Polytechnical University, Xi'an, 710072, China.

E-mail address: 89f.liao@gmail.com (F. Liao).
} 
But they are prohibitively expensive for high Reynolds wall-bounded flows due to the Reynolds number limitation [3]. Contrarily, the Reynolds-averaged Navier-Stokes (RANS) equations are cheap in costs, yet it is less accurate due to lack of unsteady information. An alternative to compromise the computational costs and the accuracy is detached eddy simulation (DES) [11] where the thin boundary layer is treated by RANS and the separated flow-field is treated by LES. In this paper, the delayed DES (DDES) [12] adopting the vorticitydependent length scale in Ref. [13] is utilized. A canonical periodic hill case [14] is validated to demonstrate DDES's capability for separated flows.

The third consideration is to find appropriately efficient and accurate way for radiated acoustics. A wave extrapolation method [15][16] is able to extrapolate the acoustics to far-field region efficiently without any dissipation and dispersion. Under such circumstances, the propagation process can be taken as a second calculation from the CFD results. FW-H equation [15] is a commonly used method and two classical strategies can be employed to obtain the numerical solutions for FW-H equations: the time domain formulation [17][18] and the frequency domain formulation [19][20]. The time domain formulation is based on the 3D Green's function whilst the frequency domain formulation can be implemented with both 2D and 3D Green's function. In this paper, a time domain formulation as well as considering the convection effect [21] is adopted. The tonal noise radiation from a laminar flow past a NACA0012 airfoil [22] is used for validation.

Finally, the radiated noise of wake-airfoil interaction is studied by investigation on the rod-airfoil problem, which can be considered as the simplified model case of the blade-vortex interaction in turbofans [23]. This problem has been experimentally investigated by Jacob et al. [24][25], and numerically studied with different approaches. The objective of this paper is, on one hand, assessing the capability of high-order CCFDM coupled with FW-H acoustic analogy for the far-field acoustics, on the other hand, providing further insight of the noise mechanism.

This paper is organized as follows. Governing equations are presented in section 2. Numerical methodologies are introduced in section 3 in detail. To validate the performance of numerical strategies, benchmark cases are provided in section 4 . Finally, section 5 investigates the radiated noise of the rod-airfoil configuration. Conclusions are drawn in section 6.

\section{Governing equations}

\subsection{Naiver-Stokes $(\mathrm{N}-\mathrm{S})$ equations}

The non-dimensional Naiver-Stokes (N-S) equations in $x, y, z$ Cartesian coordinates are

$$
\frac{\partial U(Q)}{\partial t}+\frac{\partial E(Q)}{\partial x}+\frac{\partial F(Q)}{\partial y}+\frac{\partial G(Q)}{\partial z}=\frac{M a}{R e}\left(\frac{\partial E_{v}(Q)}{\partial x}+\frac{\partial F_{v}(Q)}{\partial y}+\frac{\partial G_{v}(Q)}{\partial z}\right),
$$

where

$$
\begin{aligned}
& Q=\left(\begin{array}{c}
\rho \\
u \\
v \\
w \\
p
\end{array}\right), \quad U=\left(\begin{array}{c}
\rho \\
\rho u \\
\rho v \\
\rho w \\
\rho e
\end{array}\right), \quad E=\left(\begin{array}{c}
\rho u \\
\rho u^{2}+p \\
\rho v u \\
\rho w u \\
(\rho e+p) u
\end{array}\right), \quad F=\left(\begin{array}{c}
\rho v \\
\rho u v \\
\rho v^{2}+p \\
\rho w v \\
(\rho e+p) v
\end{array}\right), \\
& G=\left(\begin{array}{c}
\rho w \\
\rho u w \\
\rho w^{2}+p \\
(\rho e+p) w
\end{array}\right), \quad E_{v}=\left(\begin{array}{c}
0 \\
\tau_{11} \\
\tau_{12} \\
\tau_{13} \\
\varphi_{1}
\end{array}\right), \quad F_{v}=\left(\begin{array}{c}
0 \\
\tau_{21} \\
\tau_{22} \\
\tau_{23} \\
\varphi_{2}
\end{array}\right), \quad G_{v}=\left(\begin{array}{c}
0 \\
\tau_{31} \\
\tau_{32} \\
\tau_{33} \\
\varphi_{3}
\end{array}\right),
\end{aligned}
$$

where $Q$ and $U$ are primitive and conservative vector respectively. $\rho$ is density. $u, v, w$ are flow velocity components along Cartesian coordinates respectively. $p$ is pressure with $p=\rho T / \gamma$ based on the equation of state for calorically perfect gas. $T$ stands for the temperature. $\gamma=c_{p} / c_{v}$ is specific heat ratio. The total energy is $\rho e=p /(\gamma-1)+\rho\left(u^{2}+v^{2}+w^{2}\right) / 2$ where $e$ is the total energy per unit mass. The inviscid and viscous fluxes along the Cartesian coordinates are $E, F, G$ and $E_{v}, F_{v}, G_{v}$ respectively. Ma and Re are Mach number and Reynolds number. The stress tensor $\tau_{i j}$ and heat flux related term $\varphi_{i}$ in viscous fluxes $E_{v}, F_{v}$ and $G_{v}$ are

$$
\begin{aligned}
\tau_{i j} & =\left(\mu+\mu_{t}\right)\left(\frac{\partial u_{i}}{\partial x_{j}}+\frac{\partial u_{j}}{\partial x_{i}}-\frac{2}{3} \delta_{i j} \frac{\partial u_{k}}{\partial x_{k}}\right), \\
\varphi_{i} & =u_{j} \tau_{i j}+c_{p}\left(\frac{\mu}{\operatorname{Pr}}+\frac{\mu_{t}}{\operatorname{Pr}_{t}}\right) \frac{\partial T}{\partial x_{i}},
\end{aligned}
$$

where $\mu$ denotes laminar viscosity by Sutherland's formula and $\mu_{t}$ denotes turbulent viscosity according to the Boussinesq hypothesis. The specific formulations for $\mu_{t}$ will be discussed in section 2.2. The laminar and turbulent Prandtl number $\operatorname{Pr}$ and $\operatorname{Pr}_{t}$ for air is taken as 0.72 and 0.9 respectively.

All the aforementioned quantities are non-dimensionalized by

$$
\begin{aligned}
& M a=\frac{u_{\infty}^{*}}{a_{\infty}^{*}}, \quad R e=\frac{\rho_{\infty}^{*} u_{\infty}^{*} L^{*}}{\mu_{\infty}^{*}}, \quad \frac{M a}{\operatorname{Re}}=\left(\frac{\rho_{\infty}^{*} a_{\infty}^{*} L^{*}}{\mu_{\infty}^{*}}\right)^{-1}, \quad \rho=\frac{\rho^{*}}{\rho_{\infty}^{*}}, \quad p=\frac{p^{*}}{\rho_{\infty}^{*}\left(a_{\infty}^{*}\right)^{2}}, \quad T=\frac{T^{*}}{T_{\infty}^{*}}, \quad e=\frac{e^{*}}{\left(a_{\infty}^{*}\right)^{2}}, \\
& (u, v, w, a)=\frac{\left(u^{*}, v^{*}, w^{*}, a^{*}\right)}{a_{\infty}^{*}}, \quad(x, y, z)=\frac{\left(x^{*}, y^{*}, z^{*}\right)}{L^{*}}, \quad t=\frac{t^{*} a_{\infty}^{*}}{L^{*}},
\end{aligned}
$$

where the superscript * denotes the reference variables. The non-dimensional freestream parameters are 


$$
\rho_{\infty}=1, \quad p_{\infty}=\frac{1}{\gamma}, \quad T_{\infty}=1, \quad e_{\infty}=\frac{1}{\gamma(\gamma-1)}+\frac{M^{2}}{2}, \quad\left(u_{\infty}, v_{\infty}, w_{\infty}, a_{\infty}\right)=(M a, 0,0,1) .
$$

By introducing the following coordinate transformation on the stationary grid:

$$
\left\{\begin{array}{l}
x=x(\xi, \eta, \zeta), \\
y=y(\xi, \eta, \zeta), \quad J=\left|\begin{array}{lll}
x_{\xi} & x_{\eta} & x_{\zeta} \\
z=z(\xi, \eta, \zeta) . & y_{\eta} & y_{\zeta} \\
z_{\xi} & z_{\eta} & z_{\zeta}
\end{array}\right| .
\end{array}\right.
$$

Eq. (1) can be recast in the equivalent form under $\xi, \eta, \zeta$ curvilinear coordinates,

$$
\frac{\partial \hat{U}(Q)}{\partial t}+\frac{\partial \hat{E}(Q)}{\partial \xi}+\frac{\partial \hat{F}(Q)}{\partial \eta}+\frac{\partial \hat{G}(Q)}{\partial \zeta}=\frac{M a}{R e}\left(\frac{\partial \hat{E}_{v}(Q)}{\partial \xi}+\frac{\partial \hat{F}_{v}(Q)}{\partial \eta}+\frac{\partial \hat{G}_{v}(Q)}{\partial \zeta}\right),
$$

where

$$
\begin{array}{lll}
\hat{E}=J \xi_{x} E+J \xi_{y} F+J \xi_{z} G, & \hat{E}_{v}=J \xi_{x} E_{v}+J \xi_{y} F_{v}+J \xi_{z} G_{v}, \\
\hat{U}=J U, & \hat{F}=J \eta_{x} E+J \eta_{y} F+J \eta_{z} G, & \hat{F}_{v}=J \eta_{x} E_{v}+J \eta_{y} F_{v}+J \eta_{z} G_{v}, \\
& \hat{G}=J \zeta_{x} E+J \zeta_{y} F+J \zeta_{z} G, & \hat{G}_{v}=J \zeta_{x} E_{v}+J \zeta_{y} F_{v}+J \zeta_{z} G_{v},
\end{array}
$$

where the subscript notations represent derivatives, for example, notation $x_{\xi}$ stands for $\partial x / \partial \xi$. $J$ is the Jacobian of coordinate transformation.

The equivalent transformation from Eq. (1) to Eq. (7) requires the following condition to be satisfied:

$$
\begin{aligned}
& \left(J \xi_{x}\right)_{\xi}+\left(J \eta_{x}\right)_{\eta}+\left(J \zeta_{x}\right)_{\zeta}=0, \\
& \left(J \xi_{y}\right)_{\xi}+\left(J \eta_{y}\right)_{\eta}+\left(J \zeta_{y}\right)_{\zeta}=0, \\
& \left(J \xi_{z}\right)_{\xi}+\left(J \eta_{z}\right)_{\eta}+\left(J \zeta_{z}\right)_{\zeta}=0,
\end{aligned}
$$

which indicates the geometric conservation law (GCL) on stationary grid, which will be discussed in section 3.2.

\subsection{Delayed detached eddy simulation (DDES) based on Spalart-Allmaras model}

In Eq. (3), $\mu_{t}$ is obtained by

$$
\mu_{t}=f_{v 1} \rho \tilde{v}
$$

where $\tilde{v}$ is the kinetic viscosity and calculated by the Spalart-Allmaras model:

$$
\frac{\partial \tilde{v}}{\partial t}+u_{j} \frac{\partial \tilde{v}}{\partial x_{j}}=\frac{1+c_{b 2}}{\sigma}\left[\frac{\partial}{\partial x_{j}}\left((v+\tilde{v}) \frac{\partial \tilde{v}}{\partial x_{j}}\right)\right]-\frac{c_{b 2}}{\sigma}(v+\tilde{v})\left(\frac{\partial \tilde{v}}{\partial x_{j}}\right)^{2}+c_{b 1}\left(1-f_{t 2}\right) \tilde{S} \tilde{v}-\left(c_{w 1} f_{w}-\frac{c_{b 1}}{\kappa^{2}} f_{t 2}\right)\left(\frac{\tilde{v}}{d_{w}}\right)^{2},
$$

where $c_{b 1}, c_{b 2}, c_{w 1}$ and $\sigma$ are model constants. More details about $f_{v 1}, f_{t 2}$ and $f_{w}$ etc. refer to textbook by Blazek et al. [26]. $d_{w}$ is the distance to the nearest wall.

The detached eddy simulation (DES) [11][27][28][29] is realized by replacing $d_{w}$ in Eq. (11) with

$$
d_{D E S}=\min \left(d_{w}, C_{D E S} \Delta_{\max }\right),
$$

where $\Delta_{\max }=\max \left(\Delta_{x}, \Delta_{y}, \Delta_{z}\right)$ is the maximum local cell spacing and $C_{D E S}=0.65$. The modeled-stress depletion and its consequential grid-induced separation [12][30] are unavoidable issues in original DES. This means early separation may occur in boundary layer due to it that the activated $C_{D E S} \Delta_{\max }$ in Eq. (12) cannot offer enough eddy viscosity. Hence, delayed DES (DDES) technique [12] is adopted to ensure the entirely attached RANS region via a new length scale:

$$
\begin{aligned}
& d_{D D E S}=d_{w}-f_{d} \max \left(0, d_{w}-C_{D E S} \Delta_{\max }\right), \\
& f_{d}=1-\tanh \left(\left(8 r_{d}\right)^{3}\right), \\
& r_{\mathrm{d}} \equiv \frac{v_{t}+v}{\sqrt{U_{i, j} U_{i, j}} \kappa^{2} d_{w}^{2}},
\end{aligned}
$$

where $f_{d}$ equals to 0 in RANS region while approaches 1 in LES region. Standard DDES [12] tends to delay the development of physical shear layer Kelvin-Helmholtz instabilities. A vorticity-dependent length scale proposed by Chauvet et al. [13] is used to mitigate this problem:

$$
\Delta_{\omega}=\sqrt{\left(\frac{\omega_{x}}{|\boldsymbol{\omega}|}\right)^{2} \Delta y \Delta z+\left(\frac{\omega_{y}}{|\boldsymbol{\omega}|}\right)^{2} \Delta z \Delta x+\left(\frac{\omega_{z}}{|\boldsymbol{\omega}|}\right)^{2} \Delta x \Delta y},
$$

where $\omega_{x}, \omega_{y}, \omega_{z}$ represents the unit vector of local vorticity. 


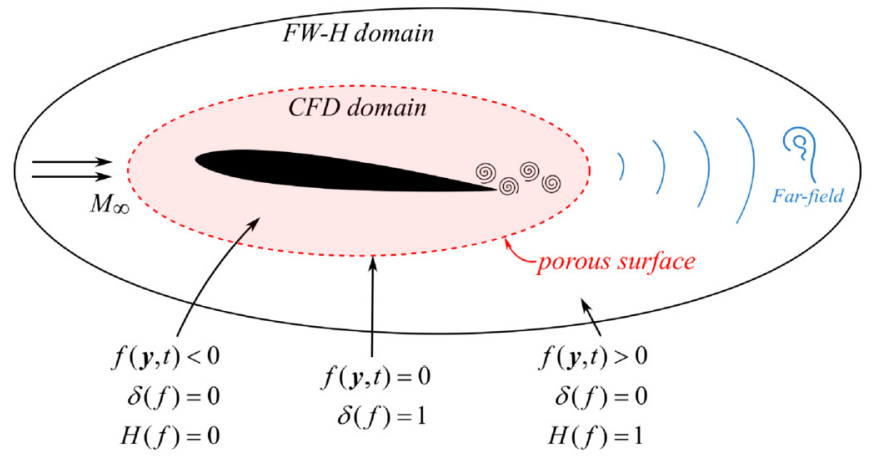

Fig. 1. Schematic diagram of the method for far-field noise prediction.

\subsection{Ffowcs Williams and Hawkings (FW-H) equation}

The convective FW-H equation [21] is solved for far-field radiated acoustics. A schematic diagram is shown in Fig. 1.

$$
\left(\frac{D^{2}}{a^{2} D t^{2}}-\nabla^{2}\right)\left[p^{\prime}(\boldsymbol{x}, t) H(f)\right]=\frac{\partial^{2}}{\partial x_{i} \partial x_{j}}\left[T_{i j} H(f)\right]-\frac{\partial}{\partial x_{i}}\left[L_{i j} n_{j} \delta(f)\right]+\frac{D}{D t}\left[\rho_{0} U_{i} n_{i} \delta(f)\right],
$$

where

$$
\begin{aligned}
\frac{D}{D t} & =\frac{\partial}{\partial t}+a M_{\infty, i} \frac{\partial}{\partial x_{i}}, & T_{i j} & =\rho u_{i} u_{j}+P_{i j}-a^{2} \rho^{\prime} \delta_{i j}, \\
L_{i j} & =\rho u_{i}\left[u_{j}-\left(v_{j}-a M_{\infty, j}\right)\right]+P_{i j}, & U_{i} & =\left(v_{i}-a M_{\infty, i}\right)+\rho\left[u_{i}-\left(v_{i}-a M_{\infty, i}\right)\right] / \rho_{0},
\end{aligned}
$$

where $a$ is the speed of sound. The convective effect is explicitly reflected by the Mach number $M_{\infty, i}$.

The solution for Eq. (15) in time domain can be written as

$$
p^{\prime}(\boldsymbol{x}, t)=\frac{1}{4 \pi} \int_{S}\left[\left(1-M_{\infty, i} R_{i}\right) \frac{\rho_{0} \dot{U}_{i} n_{i}}{R^{*}}-a M_{\infty, i} R_{i}^{*} \frac{U_{i} n_{i}}{R^{* 2}}\right]_{\tau} d S+\frac{1}{4 \pi} \int_{S}\left[\frac{1}{a} \frac{\dot{L}_{i j} n_{j} R_{i}}{R^{*}}+\frac{L_{i j} n_{j} R_{i}^{*}}{R^{* 2}}\right]_{\tau} d S,
$$

where volume integration outside the porous surface is omitted. And Eq. (17) is solved with the advanced time approach [18][31]. $\boldsymbol{y}$ and $\boldsymbol{x}$ represents the source location and the observer location respectively. $\tau$ and $t$ denote the source time and observer time. $[\cdot]_{\tau}$ means the integration is evaluated at the source time $\tau$. The dots above $\dot{L}_{i j}, \dot{U}_{i}$ stand for the temporal derivatives at the source time $\tau$, which is calculated by

$$
\tau=t-R / a,
$$

The $R, R^{*}, R_{i}, R_{i}^{*}$ appeared in Eq. (17)-(18) are given by

$$
\begin{array}{ll}
R=\left(R^{*}-M_{r}\right) / \beta^{2}, & R^{*}=\beta \sqrt{r^{2}+M_{r}^{2} / \beta^{2}}, \\
R_{i}=\left(R_{i}^{*}-M_{\infty, i}\right) / \beta^{2}, & R_{i}^{*}=\left(\beta^{2} r_{i}+M_{r} M_{\infty, i}\right) / R^{*},
\end{array}
$$

where $\beta^{2}=1-M_{\infty, i} M_{\infty, i}, M_{r}=M_{\infty, i} r_{i}$ and $r_{i}=x_{i}-y_{i}, i=1,2,3$. Details can be found in Ref. [21].

\section{Numerical methodology}

On multi-block curvilinear structured grid, solution points are located on cell centers, denoted as $(i, j, k)$. Flux points are located on face centers, denoted as $(i \pm 1 / 2, j, k),(i, j \pm 1 / 2, k)$ and $(i, j, k \pm 1 / 2)$. Grid points are located on grid nodes, denoted as $(i \pm 1 / 2, j \pm 1 / 2, k \pm 1 / 2)$.

Section 3.1 targets on the flow-dependent discretization with high-order cell-centered finite difference method (CCFDM). Section 3.2 targets on the geometry-dependent discretization with high-order cell-centered symmetric conservative metric method (CCSCMM).

\subsection{Cell-centered finite difference method (CCFDM)}

The spatial derivatives of fluxes in Eq. (7) are focused in this section. Without losing generality, the discretization of $\partial \hat{E} / \partial \xi$ is illustrated. $\hat{E}_{i}^{\prime}$ is used to denote numerical derivative of $\partial \hat{E}(Q) / \partial \xi$ along the $\xi$-coordinate where the subscript $i$ stand for the index of cell center. $\hat{E}_{i}^{\prime}$ is discretized by the face-to-cell compact difference schemes in this paper. The corresponding 4th-, 6th- and 8th-order are given by

$$
\begin{aligned}
& \text { 4th-order: } \quad \frac{1}{22} \hat{E}_{i-1}^{\prime}+\hat{E}_{i}^{\prime}+\frac{1}{22} \hat{E}_{i+1}^{\prime}=\frac{12}{11}\left(\hat{E}_{i+1 / 2}-\hat{E}_{i-1 / 2}\right), \\
& \text { 6th-order: } \quad-\frac{1}{12} \hat{E}_{i-1}^{\prime}+\hat{E}_{i}^{\prime}-\frac{1}{12} \hat{E}_{i+1}^{\prime}=\frac{16}{9}\left(\hat{E}_{i+1 / 2}-\hat{E}_{i-1 / 2}\right)-\frac{17}{36}\left(\hat{E}_{i+1}-\hat{E}_{i-1}\right), \\
& \text { 8th-order: } \quad-\frac{3}{20} \hat{E}_{i-1}^{\prime}+\hat{E}_{i}^{\prime}-\frac{3}{20} \hat{E}_{i+1}^{\prime}=2\left(\hat{E}_{i+1 / 2}-\hat{E}_{i-1 / 2}\right)-\frac{61}{100}\left(\hat{E}_{i+1}-\hat{E}_{i-1}\right)-\frac{2}{75}\left(\hat{E}_{i+3 / 2}-\hat{E}_{i-3 / 2}\right) .
\end{aligned}
$$




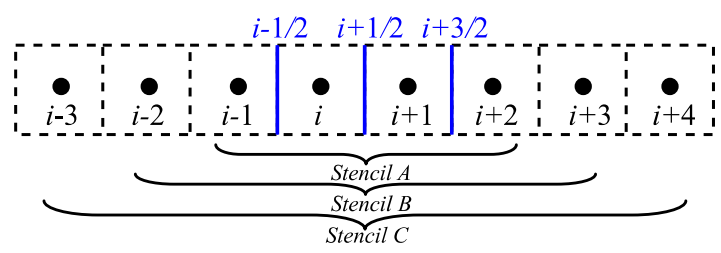

Fig. 2. Schematic diagram of the stencil for Opt4, Opt6 and Opt8.

Table 1

Coefficients of schemes in Eq. (22) related to $\xi$ and $\eta$.

\begin{tabular}{|c|c|c|c|c|c|c|c|}
\hline & Stencil & $\beta_{-1}, \beta_{1}$ & $c_{-3}, c_{4}$ & $c_{-2}, c_{3}$ & $c_{-1}, c_{2}$ & $c_{0}, c_{1}$ & $T(\xi, \eta, k)$ \\
\hline Opt4 & A & $\frac{\eta \mp \xi}{2}$ & 0 & 0 & $\frac{-1+3 \eta \mp 2 \xi}{16}$ & $\frac{9+5 \eta \mp 10 \xi}{16}$ & Eq. (23) \\
\hline Opt6 & B & $\frac{\eta \mp \xi}{2}$ & 0 & $\frac{3-5 \eta \pm 2 \xi}{256}$ & $\frac{-25+63 \eta \mp 42 \xi}{256}$ & $\frac{5(15+7 \eta \mp 14 \xi)}{128}$ & Eq. (24) \\
\hline Opt8 & C & $\frac{\eta \mp \xi}{2}$ & $\frac{-5+7 \eta \mp 2 \xi}{2048}$ & $\frac{49-75 \eta \pm 30 \xi}{2048}$ & $\frac{7(-35+81 \eta \mp 54 \xi)}{2048}$ & $\frac{175(7+3 \eta \mp 6 \xi)}{2048}$ & Eq. (25) \\
\hline
\end{tabular}

The left-hand side derivatives $\hat{E}_{i}^{\prime}$ etc. in Eq. (20) can be acquired by tridiagonal inversion through the Thomas algorithm assuming the right-hand side terms are known. The right-hand side fluxes in Eq. (20) on face centers are evaluated by a Riemann flux solver, for instance $\hat{E}_{i+1 / 2}$ with Roe scheme is given by

$$
\hat{E}_{i+1 / 2}=\operatorname{Roe}\left(Q_{i+1 / 2}^{L}, Q_{i+1 / 2}^{R}, J \xi_{x, i+1 / 2}, J \xi_{y, i+1 / 2}, J \xi_{z, i+1 / 2}\right),
$$

where the coordinate transformation metrics will be discussed in the next section. $Q_{i+1 / 2}^{L}$ and $Q_{i+1 / 2}^{R}$ are the interpolated primitive variables to the left and right of the face center $i+1 / 2$, respectively. To improve the dispersion and dissipation properties of the whole method, three optimized tridiagonal compact interpolations (Opt4, Opt6 and Opt8) are proposed. Fig. 2 shows the respective discretization stencils (A, B and C) for Opt4, Opt6 and Opt8. A general formulation for $Q_{i+1 / 2}^{L}$ and $Q_{i+1 / 2}^{R}$ is

$$
\beta_{-1} Q_{i-1 / 2}+Q_{i+1 / 2}+\beta_{1} Q_{i+3 / 2}=c_{-3} Q_{i-3}+c_{-2} Q_{i-2}+c_{-1} Q_{i-1}+c_{0} Q_{i}+c_{1} Q_{i+1}+c_{2} Q_{i+2}+c_{3} Q_{i+3}+c_{4} Q_{i+4},
$$

where the corresponding coefficients are given in Table 1. In each of the Opt4, Opt6 and Opt8 schemes, two degrees of freedom, denoted by $\xi$ and $\eta$, are used for dissipation and dispersion optimization.

By analyzing the spectral behavior in wavenumber space, the transfer function $T(\xi, \eta, k)$ for Eq. (22), where $k=\omega \Delta x$, are obtained. The transfer function for Opt4 is

$$
T(\xi, \eta, k)_{O p t 4}=\frac{\left[\left(\frac{1}{8}(-1+3 \eta) \cos \left(\frac{3}{2} k\right)+\frac{1}{8}(9+5 \eta) \cos \left(\frac{1}{2} k\right)\right)+i\left(\frac{1}{4} \xi \sin \left(\frac{3}{2} k\right)+\frac{5}{4} \xi \sin \left(\frac{1}{2} k\right)\right)\right]}{(\eta \cos (k)+1)+i(\xi \sin (k))} .
$$

The transfer function for Opt6 is

$$
T(\xi, \eta, k)_{O p t 6}=\frac{\left[\begin{array}{l}
\frac{1}{128}(3-5 \eta) \cos \left(\frac{5}{2} k\right)+\frac{1}{128}(-25+63 \eta) \cos \left(\frac{3}{2} k\right) \\
+\frac{5}{64}(15+7 \eta) \cos \left(\frac{1}{2} k\right)
\end{array}\right)+i\left(\begin{array}{c}
-\frac{1}{64} \xi \sin \left(\frac{5}{2} k\right)+\frac{21}{64} \xi \sin \left(\frac{3}{2} k\right) \\
35 \\
+\frac{35}{32} \xi \sin \left(\frac{1}{2} k\right)
\end{array}\right)}{(\eta \cos (k)+1)+i(\xi \sin (k))} .
$$

The transfer function for Opt8 is

$$
\begin{aligned}
T(\xi, \eta, k) \text { Opt8 } & {\left.\left[\begin{array}{l}
\frac{1}{1024}(-5+7 \eta) \cos \left(\frac{7}{2} k\right)+\frac{1}{1024}(49-75 \eta) \cos \left(\frac{5}{2} k\right)+ \\
\frac{1}{1024}(-245+567 \eta) \cos \left(\frac{3}{2} k\right)+\frac{1}{1024}(1225+525 \eta) \cos \left(\frac{1}{2} k\right)
\end{array}\right)+i\left(\begin{array}{l}
\frac{1}{512} \xi \sin \left(\frac{7}{2} k\right)-\frac{15}{512} \xi \sin \left(\frac{5}{2} k\right)+ \\
\frac{189}{512} \xi \sin \left(\frac{3}{2} k\right)+\frac{525}{512} \xi \sin \left(\frac{1}{2} k\right)
\end{array}\right)\right] } \\
& =\frac{(\eta \cos (k)+1)+i(\xi \sin (k))}{} .
\end{aligned}
$$

For a given $\xi$ and $\eta$, the transfer function $T(\xi, \eta, k)$ consists of two components: the real part $T_{\Re}(\xi, \eta, k)$ and the imaginary part $T_{\Im}(\xi, \eta, k) . T_{\Re}(\xi, \eta, k)$ is associated with dispersion property while $T_{\Im}(\xi, \eta, k)$ is related to dissipation property.

The optimized $\xi$ and $\eta$ can be found to eliminate the spectral error in wavenumber domain. Specifically, the following integrated dispersion and dissipation error function should reach its minimum value.

$$
\left(\xi_{\text {opt }}, \eta_{\text {opt }}\right)=\arg \min _{(\xi, \eta)}\left\{\int_{0}^{k_{c}}\left[0.5\left|T_{\Re}(\xi, \eta, k)-1\right|+0.5\left|T_{\Im}(\xi, \eta, k)-0\right|\right] d k\right\}, \quad \text { s.t. }\left\{\begin{array}{l}
T_{\Re}(\xi, \eta, k)-1<\varepsilon_{\Re} \\
\int_{0}^{k_{c}}\left|T_{\Im}(\xi, \eta, k)-0\right| d k<\varepsilon_{\Im} \\
\xi_{\min } \leq \xi \leq \xi_{\max } \\
\eta_{\min } \leq \eta \leq \eta_{\max }
\end{array}\right.
$$


Table 2

The optimization parameters for Opt4, Opt6 and Opt8 respectively.

\begin{tabular}{|c|c|c|c|c|c|c|c|c|c|}
\hline & $k_{c}$ & $\varepsilon_{\Re}$ & $\varepsilon_{\Im}$ & $\xi_{\min }$ & $\xi_{\max }$ & $\eta_{\min }$ & $\eta_{\max }$ & $\xi_{\text {opt }}$ & $\eta_{o p t}$ \\
\hline Opt4 & $2 \pi / 3$ & $2 \%$ & $\frac{1}{3} \int_{0}^{k_{c}}\left|T_{\Im}^{U I 5}(k)\right| d k$ & $-2 / 5$ & 0 & $3 / 5$ & 1 & -0.104 & 0.731 \\
\hline Opt6 & $5 \pi / 6$ & $1 \%$ & $\frac{1}{3} \int_{0}^{k_{c}}\left|T_{\Im}^{U I 7}(k)\right| d k$ & $-2 / 7$ & 0 & $5 / 7$ & 1 & -0.080 & 0.810 \\
\hline Opt8 & $6 \pi / 7$ & $0.5 \%$ & $\frac{1}{3} \int_{0}^{k_{c}}\left|T_{\Im}^{U I 9}(k)\right| d k$ & $-2 / 9$ & 0 & $7 / 9$ & 1 & -0.062 & 0.848 \\
\hline
\end{tabular}
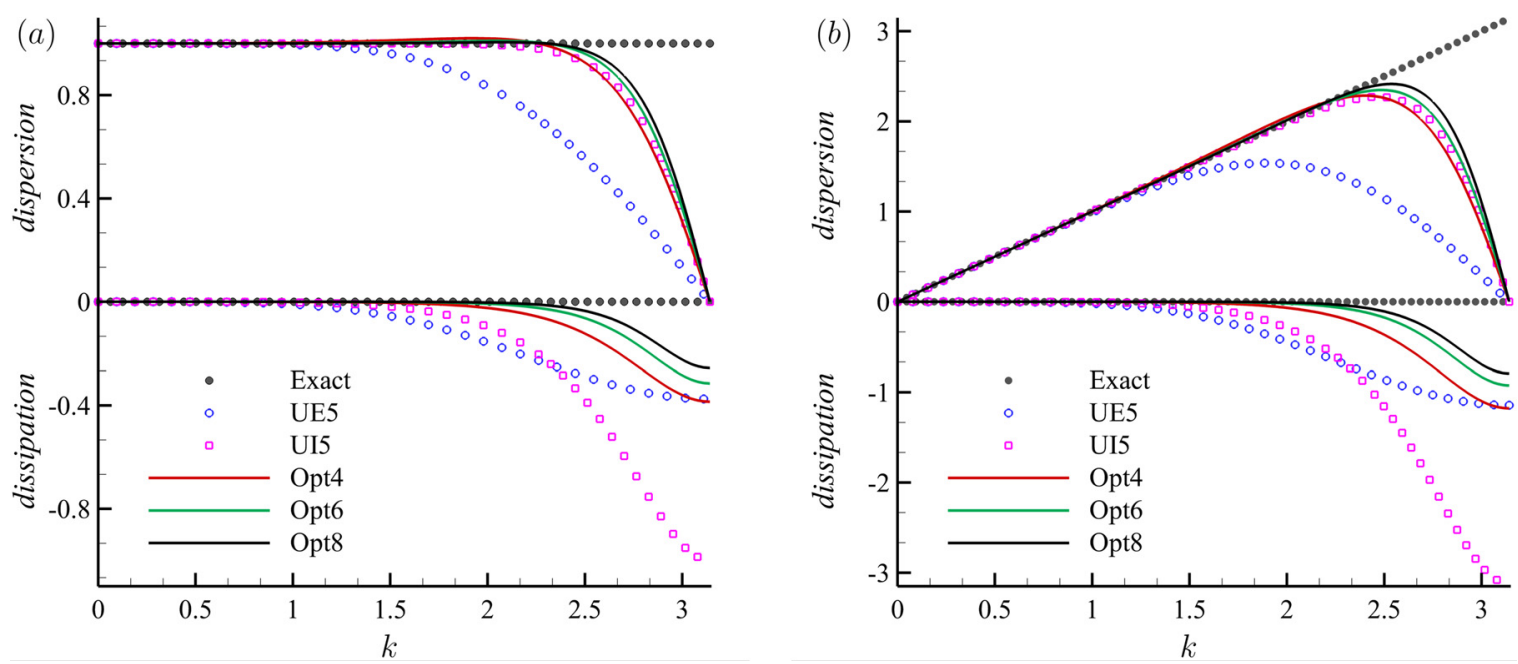

Fig. 3. Spectral properties. (a): Interpolations (Eq. (22)); (b): Interpolations (Eq. (22)) coupled with the corresponding same order difference schemes (Eq. (20)).

where $k_{c}$ is the cut-off wavenumber below which the optimization procedure is valid. $\varepsilon_{\Re}$ and $\varepsilon_{\Im}$ are the constraint thresholds for dispersion and dissipation error respectively. The optimization parameters and the optimized $\xi$ and $\eta$ are given in Table 2 where $T_{\Im}^{U I 5}(k)$, $T_{\Im}^{U I 7}(k)$ and $T_{\Im}^{U I 9}(k)$ are the imaginary parts of the transfer functions of 5th-order, 7th-order and 9th-order upwind tridiagonal compact schemes (denoted as UI5, UI7 and UI9 respectively), which can be obtained by setting $(\xi, \eta)$ into $(-2 / 5,3 / 5),(-2 / 7,5 / 7)$ and $(-2 / 9,7 / 9)$ in Eq. (22) and Table 1, respectively.

The spectral properties of Opt4, Opt6 and Opt8 are shown in Fig. 3. And the comparisons are also made with a 5th-order upwind explicit scheme (UE5) and a 5th-order upwind compact scheme (UI5). It can be seen that Opt4, Opt6 and Opt8 improve the spectral properties dramatically.

$$
\begin{aligned}
& \text { (Upwind - Explicit - 5/UE5) }: Q_{i+1 / 2}=\frac{3}{128} Q_{i-2}-\frac{5}{32} Q_{i-1}+\frac{45}{64} Q_{i}+\frac{15}{32} Q_{i+1}-\frac{5}{128} Q_{i+2}, \\
& \text { (Upwind - Implicit - 5/UI5) }: \frac{1}{2} Q_{i-1 / 2}+Q_{i+1 / 2}+\frac{1}{10} Q_{i+3 / 2}=\frac{1}{10} Q_{i-1}+Q_{i}+\frac{1}{2} Q_{i+1} .
\end{aligned}
$$

\subsection{Cell-centered symmetric conservative metric method (CCSCMM)}

The coordinate transformation metrics in Eq. (8) and Eq. (21) are calculated by [4][5]:

$$
\begin{aligned}
& J \xi_{x}=\frac{1}{2}\left[\left(J \xi_{x}\right)^{S 1}+\left(J \xi_{x}\right)^{S 2}\right], J \xi_{y}=\frac{1}{2}\left[\left(J \xi_{y}\right)^{S 1}+\left(J \xi_{y}\right)^{S 2}\right], J \xi_{z}=\frac{1}{2}\left[\left(J \xi_{z}\right)^{S 1}+\left(J \xi_{z}\right)^{S 2}\right], \\
& J \eta_{x}=\frac{1}{2}\left[\left(J \eta_{x}\right)^{S 1}+\left(J \eta_{x}\right)^{S 2}\right], J \eta_{y}=\frac{1}{2}\left[\left(J \eta_{y}\right)^{S 1}+\left(J \eta_{y}\right)^{S 2}\right], J \eta_{z}=\frac{1}{2}\left[\left(J \eta_{z}\right)^{S 1}+\left(J \eta_{z}\right)^{S 2}\right], \\
& J \zeta_{x}=\frac{1}{2}\left[\left(J \zeta_{x}\right)^{S 1}+\left(J \zeta_{x}\right)^{S 2}\right], J \zeta_{y}=\frac{1}{2}\left[\left(J \zeta_{y}\right)^{S 1}+\left(J \zeta_{y}\right)^{S 2}\right], J \zeta_{z}=\frac{1}{2}\left[\left(J \zeta_{z}\right)^{S 1}+\left(J \zeta_{z}\right)^{S 2}\right],
\end{aligned}
$$

where

$$
\begin{aligned}
& \left(J \xi_{x}\right)^{S 1}=\left(y_{\eta} z\right)_{\zeta}-\left(y_{\zeta} z\right)_{\eta},\left(J \xi_{y}\right)^{S 1}=\left(z_{\eta} x\right)_{\zeta}-\left(z_{\zeta} x\right)_{\eta},\left(J \xi_{z}\right)^{S 1}=\left(x_{\eta} y\right)_{\zeta}-\left(x_{\zeta} y\right)_{\eta}, \\
& \left(J \eta_{x}\right)^{S 1}=\left(y_{\zeta} z\right)_{\xi}-\left(y_{\xi} z\right)_{\zeta},\left(J \eta_{y}\right)^{S 1}=\left(z_{\zeta} x\right)_{\xi}-\left(z_{\xi} x\right)_{\zeta},\left(J \eta_{z}\right)^{S 1}=\left(x_{\zeta} y\right)_{\xi}-\left(x_{\xi} y\right)_{\zeta}, \\
& \left(J \zeta_{x}\right)^{S 1}=\left(y_{\xi} z\right)_{\eta}-\left(y_{\eta} z\right)_{\xi},\left(J \zeta_{y}\right)^{S 1}=\left(z_{\xi} x\right)_{\eta}-\left(z_{\eta} x\right)_{\xi},\left(J \zeta_{z}\right)^{S 1}=\left(x_{\xi} y\right)_{\eta}-\left(x_{\eta} y\right)_{\xi}, \\
& \left(J \xi_{x}\right)^{S 2}=\left(y z_{\zeta}\right)_{\eta}-\left(y z_{\eta}\right)_{\zeta},\left(J \xi_{y}\right)^{S 2}=\left(z x_{\zeta}\right)_{\eta}-\left(z x_{\eta}\right)_{\zeta},\left(J \xi_{z}\right)^{S 2}=\left(x y_{\zeta}\right)_{\eta}-\left(x y_{\eta}\right)_{\zeta}, \\
& \left(J \eta_{x}\right)^{S 2}=\left(y z_{\xi}\right)_{\zeta}-\left(y z_{\zeta}\right)_{\xi},\left(J \eta_{y}\right)^{S 2}=\left(z x_{\xi}\right)_{\zeta}-\left(z x_{\zeta}\right)_{\xi},\left(J \eta_{z}\right)^{S 2}=\left(x y_{\xi}\right)_{\zeta}-\left(x y_{\zeta}\right)_{\xi}, \\
& \left(J \zeta_{x}\right)^{S 2}=\left(y z_{\eta}\right)_{\xi}-\left(y z_{\xi}\right)_{\eta},\left(J \zeta_{y}\right)^{S 2}=\left(z x_{\eta}\right)_{\xi}-\left(z x_{\xi}\right)_{\eta},\left(J \zeta_{z}\right)^{S 2}=\left(x y_{\eta}\right)_{\xi}-\left(x y_{\xi}\right)_{\eta} .
\end{aligned}
$$



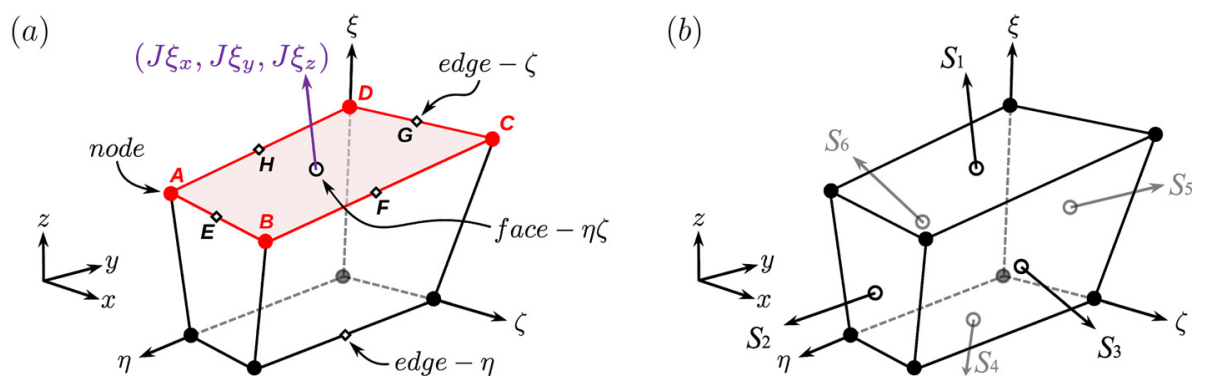

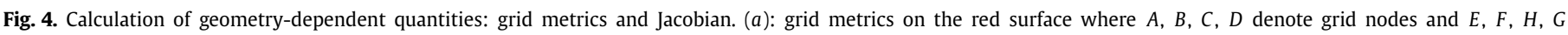

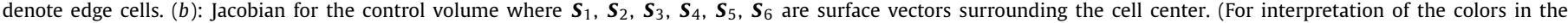
figure(s), the reader is referred to the web version of this article.)

As illustration, term $\left(J \xi_{x}\right)^{S 1}$ is discretized by

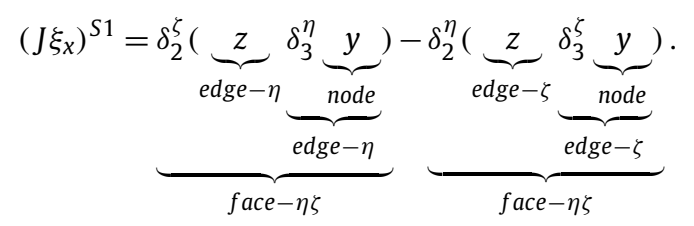

Jacobian $J$ is discretized by

$$
\begin{aligned}
& J=\frac{1}{3}\{\underbrace{\left\{\delta_{1}^{\xi}[\underbrace{x}_{\text {face }-\eta \zeta} \underbrace{\left(J \xi_{x}\right)}_{\text {face }-\eta \zeta}+\underbrace{y}_{\text {face }-\eta \zeta} \underbrace{\left(J \xi_{y}\right)}_{\text {face }-\eta \zeta}+\underbrace{z}_{\text {face }-\eta \zeta \text { face }-\eta \zeta} \underbrace{\left(J \xi_{z}\right)}]\right.}_{\text {cell }-\xi \eta \zeta} \\
& +\underbrace{\delta_{1}^{\eta}[\underbrace{x}_{\text {face }-\xi \zeta} \underbrace{\left(J \eta_{x}\right)}_{\text {face }-\xi \zeta}+\underbrace{y}_{\text {face }-\xi \zeta \text { face }-\xi \zeta} \underbrace{\left(J \eta_{y}\right)}_{\text {face }-\xi \zeta \text { face }-\xi \zeta}+\underbrace{\left(J \eta_{z}\right)}]}_{\text {cell }-\xi \eta \zeta} \\
& +\underbrace{\left.\delta_{1}^{\zeta}[\underbrace{x}_{\text {face }-\xi \eta} \underbrace{\left(J \zeta_{x}\right)}_{\text {face }-\xi \eta}+\underbrace{y}_{\text {face }-\xi \eta} \underbrace{\left(J \zeta_{y}\right)}_{\text {face }-\xi \eta}+\underbrace{z}_{\text {face }-\xi \eta} \underbrace{\left(J \zeta_{z}\right)}_{\text {face }-\xi \eta}]\right\} .}_{\text {cell }-\xi \eta \zeta}
\end{aligned}
$$

Eqs. (30)-(31) involve three categories of difference operators, which are node-to-edge difference operator $\delta_{3}$; edge-to-face difference operator $\delta_{2}$ and face-to-cell difference operator $\delta_{1}$, and two kinds of interpolation operators, which are node-to-edge interpolation and edge-to-face interpolation.

For clarity, the derivatives and interpolations in Eqs. (30)-(31) will be demonstrated with 2nd-order schemes:

$$
\begin{aligned}
\delta(\bullet)_{i} & =(\bullet)_{i+1 / 2}-(\bullet)_{i-1 / 2} \\
(\bullet)_{i} & =\frac{1}{2}\left[(\bullet)_{i+1 / 2}+(\bullet)_{i-1 / 2}\right] .
\end{aligned}
$$

And, on the red surface in Fig. $4(a),\left(J \xi_{x}\right)^{S 1}$ is given by

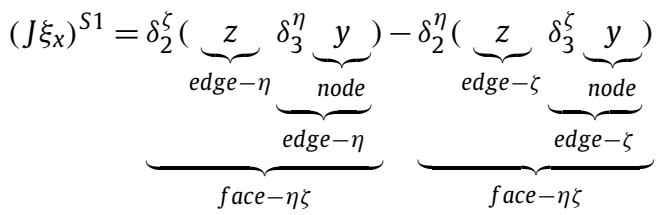

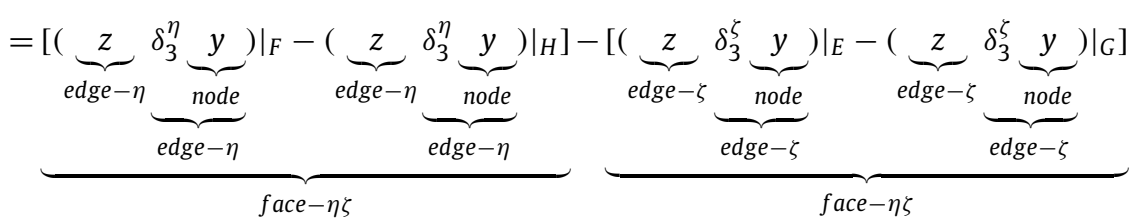

$$
\begin{aligned}
& =\left[\frac{z_{B}+z_{C}}{2}\left(y_{B}-y_{C}\right)-\frac{z_{A}+z_{D}}{2}\left(y_{A}-y_{D}\right)\right]-\left[\frac{z_{A}+z_{B}}{2}\left(y_{B}-y_{A}\right)-\frac{z_{C}+z_{D}}{2}\left(y_{C}-y_{D}\right)\right] \\
& =\frac{1}{2}\left[\left(y_{A}-y_{C}\right)\left(z_{B}-z_{D}\right)-\left(y_{B}-y_{D}\right)\left(z_{A}-z_{C}\right)\right] \text {. }
\end{aligned}
$$


The similar relations also hold for $\left(J \xi_{y}\right)^{S 1}$ and $\left(J \xi_{z}\right)^{S 1}$ :

$$
\begin{aligned}
& \left(J \xi_{y}\right)^{S 1}=\frac{1}{2}\left[\left(z_{A}-z_{C}\right)\left(x_{B}-x_{D}\right)-\left(z_{B}-z_{D}\right)\left(x_{A}-x_{C}\right)\right], \\
& \left(J \xi_{z}\right)^{S 1}=\frac{1}{2}\left[\left(x_{A}-y_{C}\right)\left(y_{B}-y_{D}\right)-\left(x_{B}-x_{D}\right)\left(y_{A}-y_{C}\right)\right] .
\end{aligned}
$$

Combining Eq. (33) and (34) together, we have

$$
\left(J \xi_{x}, J \xi_{y}, J \xi_{z}\right)^{S 1}=\frac{1}{2} \overrightarrow{C A} \times \overrightarrow{D B}=\left(S_{x}, S_{y}, S_{z}\right)=\mathbf{S}_{1},
$$

which means the 2nd-order metrics in CCFDM is equivalent to the surface vector of CCFVM in numeric.

A schematic diagram for the calculation of Jacobian is shown in Fig. 4 (b). Using divergence theorem, Eq. (31) can be further rewritten as

$$
\begin{aligned}
& J=\frac{1}{3}\{\underbrace{\left\{\delta_{1}^{\xi}[\underbrace{(x, y, z) \cdot\left(J \xi_{x}, J \xi_{y}, J \xi_{z}\right)}_{\text {face }-\eta \zeta}]\right.}_{\text {cell }-\xi \eta \zeta}+\underbrace{\delta_{1}^{\eta}[\underbrace{\left[(x, y, z) \cdot\left(J \eta_{x}, J \eta_{y}, J \eta_{z}\right)\right.}_{\text {face- } \xi \zeta}]}_{\text {cell }-\xi \eta \zeta}+\underbrace{\delta_{1}^{\zeta}[\underbrace{(x, y, z) \cdot\left(J \zeta_{x}, J \zeta_{y}, J \zeta_{z}\right)}_{\text {face- } \xi \eta}]}_{\text {cell }-\xi \eta \zeta}\} \\
& =\frac{1}{3}\left\{\left[(x, y, z){ }_{1} \mathbf{S}_{1}-(x, y, z){ }_{4} \mathbf{S}_{4}\right]+\left[(x, y, z){ }_{2} \mathbf{S}_{2}-(x, y, z)_{5} \mathbf{S}_{5}\right]+\left[(x, y, z){ }_{3} \mathbf{S}_{3}-(x, y, z){ }_{6} \mathbf{S}_{6}\right]\right\} \\
& =\frac{1}{3} \iint_{\partial V}(x, y, z) d \mathbf{S}=\frac{1}{3} \iiint_{V} \nabla \cdot(x, y, z) d V=\frac{1}{3} \iiint_{V}\left(\frac{\partial x}{\partial x}+\frac{\partial y}{\partial y}+\frac{\partial z}{\partial z}\right) d V \\
& =V
\end{aligned}
$$

which means the 2nd-order Jacobian in CCFDM is equivalent to the volume of the control volume in CCFVM.

By replacing the 2nd-order approximations (Eq. (32)) in Eqs. (33)-(36) with their corresponding high-order counterparts, the high-order grid metrics and Jacobian are acquired. The accuracy consistency is guarantee by using 4th-, 6th-, and 8th-order derivatives, which are same with that in Eq. (20). And the following 4th-, 6th-, and 8th-order interpolations are given by

$$
\begin{aligned}
& \text { 4th-order: } \frac{1}{6}(\bullet)_{i-1}+(\bullet)_{i}+\frac{1}{6}(\bullet)_{i+1}=\frac{2}{3}\left[(\bullet)_{i+1 / 2}+(\bullet)_{i-1 / 2}\right] \\
& \text { 6th-order: } \frac{3}{10}(\bullet)_{i-1}+(\bullet)_{i}+\frac{3}{10}(\bullet)_{i+1}=\frac{3}{4}\left[(\bullet)_{i+1 / 2}+(\bullet)_{i-1 / 2}\right]+\frac{1}{20}\left[(\bullet)_{i+3 / 2}+(\bullet)_{i-3 / 2}\right] \\
& \text { 8th-order: } \frac{5}{14}(\bullet)_{i-1}+(\bullet)_{i}+\frac{5}{14}(\bullet)_{i+1}=\frac{25}{32}\left[(\bullet)_{i+1 / 2}+(\bullet)_{i-1 / 2}\right]+\frac{5}{64}\left[(\bullet)_{i+3 / 2}+(\bullet)_{i-3 / 2}\right]-\frac{1}{448}\left[(\bullet)_{i+5 / 2}+(\bullet)_{i-5 / 2}\right] .
\end{aligned}
$$

In combination of section 3.1 and section 3.2, the numerical discretization of flow-dependent variable and geometry-dependent variable are both calculated with high-order accuracy.

\subsection{Time marching technique}

For inviscid cases, the set of ODEs are advanced by explicit TVD Runge-Kutta scheme [32]. The maximally allowable time step is specified according to the CFL number less than 1. With regard to wall-bounded viscous flows with high Reynolds number, implicit technique provides an appropriate alternative due to large allowable CFL number. The diagonalized diagonal dominant alternating direction implicit (D3ADI) scheme [33] is adopted for the rod-airfoil configuration.

\section{Validations}

\subsection{Isentropic vortex problem}

This problem [34] is utilized to demonstrate the accuracy of Opt4, Opt6 and Opt8. An isentropic vortex initially locates at $\left(x_{c}, y_{c}\right)=$ $(0,0)$ with the following given conditions.

$$
\begin{aligned}
& (\delta u, \delta v)=\frac{\beta}{2 \pi} e^{\left(\frac{1-r^{2}}{2}\right)}\left[-\left(y-y_{c}\right),\left(x-x_{c}\right)\right], \delta T=-\frac{(\gamma-1) \beta^{2}}{8 \gamma \pi^{2}} e^{\left(1-r^{2}\right)}, \rho=T^{\frac{1}{\gamma-1}}, \\
& P=\rho T, r=\sqrt{\left(x-x_{c}\right)^{2}+\left(y-y_{c}\right)^{2}} .
\end{aligned}
$$

Two kinds of grids are focused: uniform grid and wavy grid. The method for generating these grids can be found in Ref. [35]. Fig. 5 evidences that the vortex is well preserved on both uniform and wavy grids.

To quantitatively evaluate the numerical error, six gradually finer grids are adopted, containing $60 \times 60,80 \times 80,100 \times 100$, $120 \times 120,140 \times 140$ and $160 \times 160$ cells respectively. Error on a specific grid is defined by 
(a)

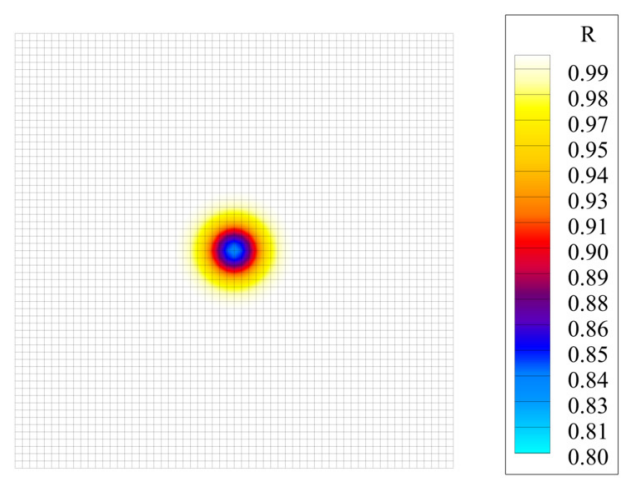

(b)

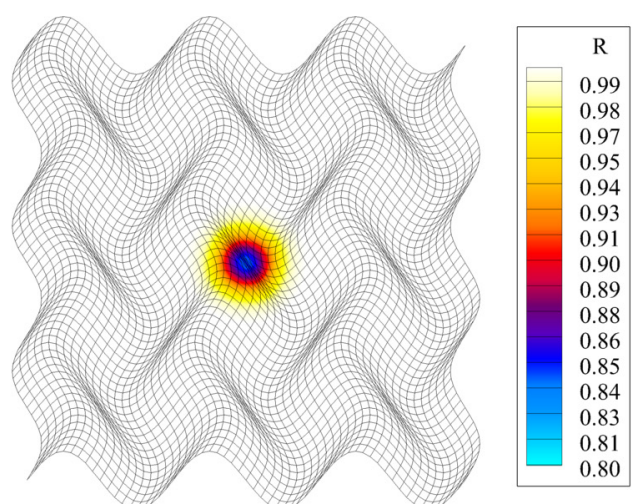

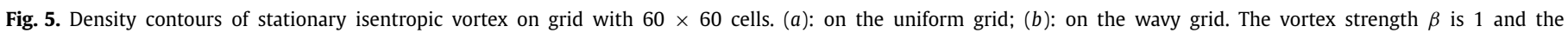
simulation ends at $t=12$.
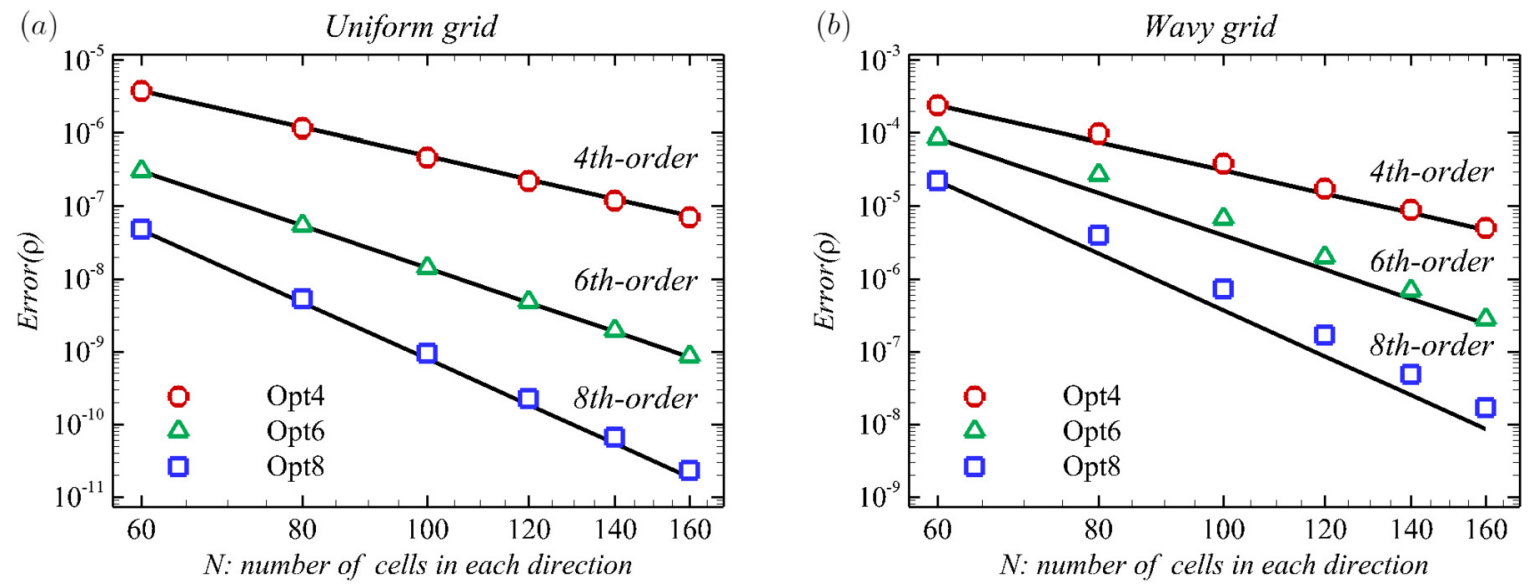

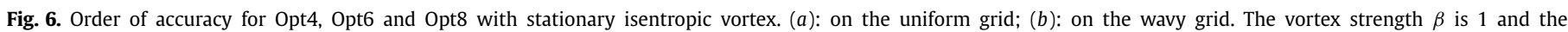
simulation ends at $t=12$.
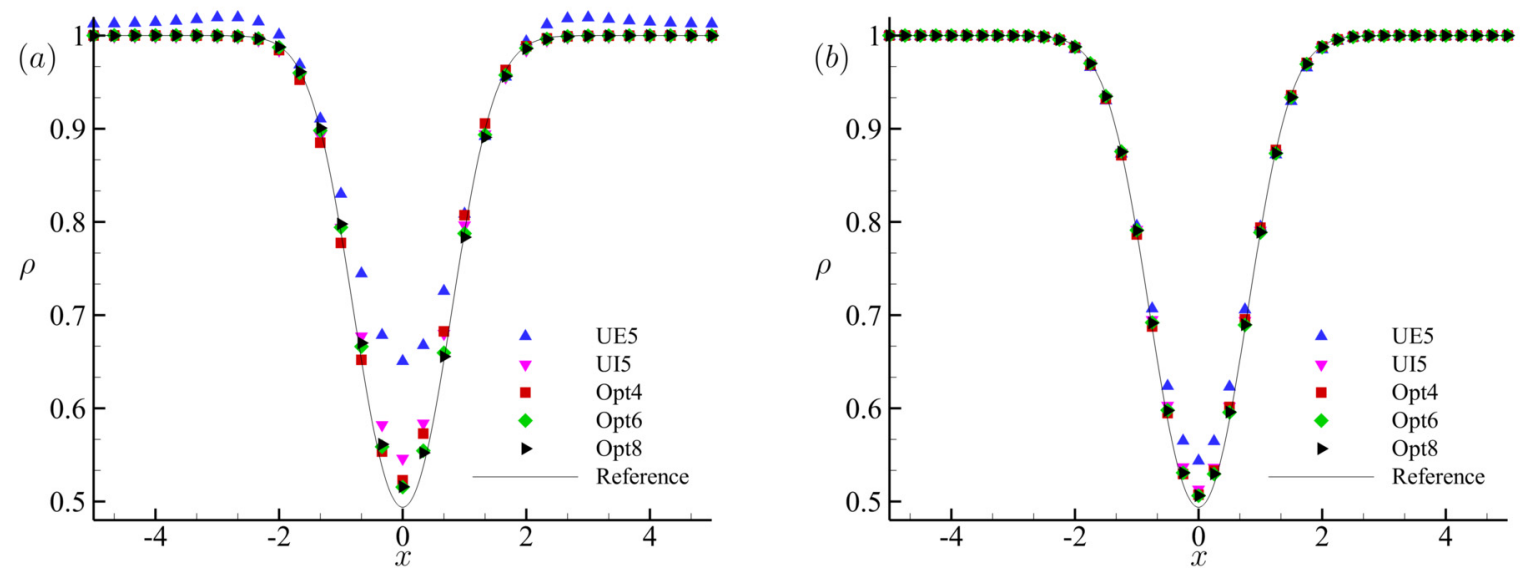

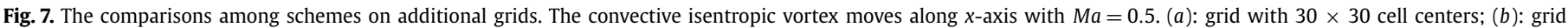
with $40 \times 40$ cell centers. The vortex strength $\beta$ is 5 and the simulation stops at $t=100$.

$$
\operatorname{Error}(\rho)=\sqrt{\sum_{i=1}^{N} \sum_{j=1}^{N}\left[\rho_{i, j}(t)-\rho_{i, j}(0)\right]^{2} / N^{2}}
$$

Fig. 6 indicates high-order accuracy can be preserved on both uniform and wavy grids.

Additionally, the performance of various schemes in preserving a stronger moving vortex on two tiny grids after a longer time simulation is also compared in Fig. 7. The optimized schemes behave less dissipatively. 

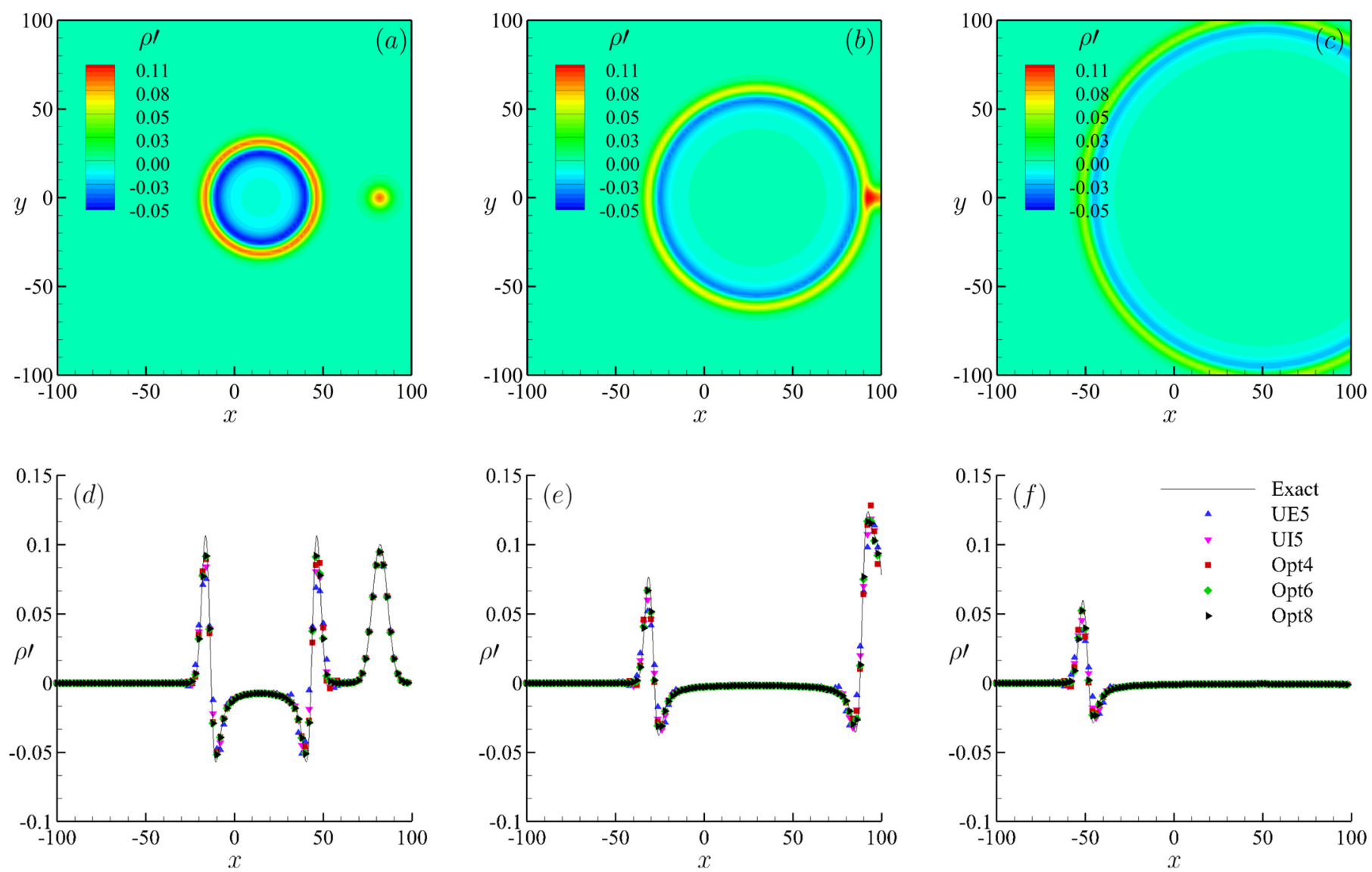

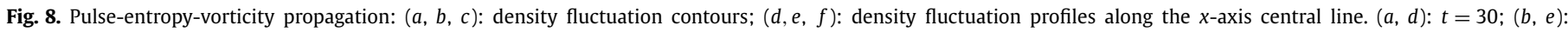
$t=60 ;(c, f): t=100$.

\subsection{Pulse-entropy-vorticity propagation}

The pulse-entropy-vorticity propagation problem [36] consists of an acoustic wave, a vorticity wave and an entropy wave propagating in a uniform flow with $M a=0.5$. The initial perturbation variables are given by

$$
\left\{\begin{array}{l}
p^{\prime}=\varepsilon \exp \left[-(\ln 2)\left(x^{2}+y^{2}\right) / 9\right], \\
\rho^{\prime}=\varepsilon \exp \left[-(\ln 2)\left(x^{2}+y^{2}\right) / 9\right]+0.1 \varepsilon \exp \left[-(\ln 2)\left((x-67)^{2}+y^{2}\right) / 25\right], \\
u^{\prime}=0.04 \varepsilon y \exp \left[-(\ln 2)\left((x-67)^{2}+y^{2}\right) / 25\right], \\
v^{\prime}=-0.04 \varepsilon(x-67) \exp \left[-(\ln 2)\left((x-67)^{2}+y^{2}\right) / 25\right],
\end{array}\right.
$$

where $\varepsilon=0.001$ is used to linearize Euler equations. Similar post-processing is taken for case 4.3 and 4.4 . The presented results are multiplied by $1 / \varepsilon=1000$ to compare with the analytical solution in Fig. 8. The computational domain is $[-100,100] \times[-100,100]$ on a uniform grid with $\Delta x=\Delta y=2$. It is clear that UE5 produces noticeable dispersion and dissipation error. With UI5, the dissipation error is eliminated but the numerical dispersive error has still not been improved. Opt6 and Opt8 give solutions closest to the exact solutions.

\subsection{Scattering of a sound wave from a solid wall}

This case [36] is to show the capability of schemes in capturing a propagating wave with $M a=0.5$ radiated by a solid wall. The computational domain is $[-100,100] \times[0,200]$ with the bottom boundary is treated as a solid wall. The grid is uniformly spaced with $\Delta x=\Delta y=2$. An initial acoustic pulse is given by

$$
\left\{\begin{array}{l}
p^{\prime}=\varepsilon \exp \left[-(\ln 2)\left(x^{2}+(y-25)^{2}\right) / 25\right], \\
\rho^{\prime}=\varepsilon \exp \left[-(\ln 2)\left(x^{2}+(y-25)^{2}\right) / 25\right], \\
u^{\prime}=v^{\prime}=0
\end{array}\right.
$$

where $\varepsilon=0.001$. Fig. 9 shows that the results produced by the compact schemes agree well with the analytical solutions.

\subsection{Scattering of sound wave from multiple circular cylinders}

This case brought up in the fourth CAA workshop [37] is to test the capability of numerical schemes in the presence of complex geometries. Both two and three-cylinder cases are investigated. Since the configurations are symmetric about $y=0$ plane, only the upper 

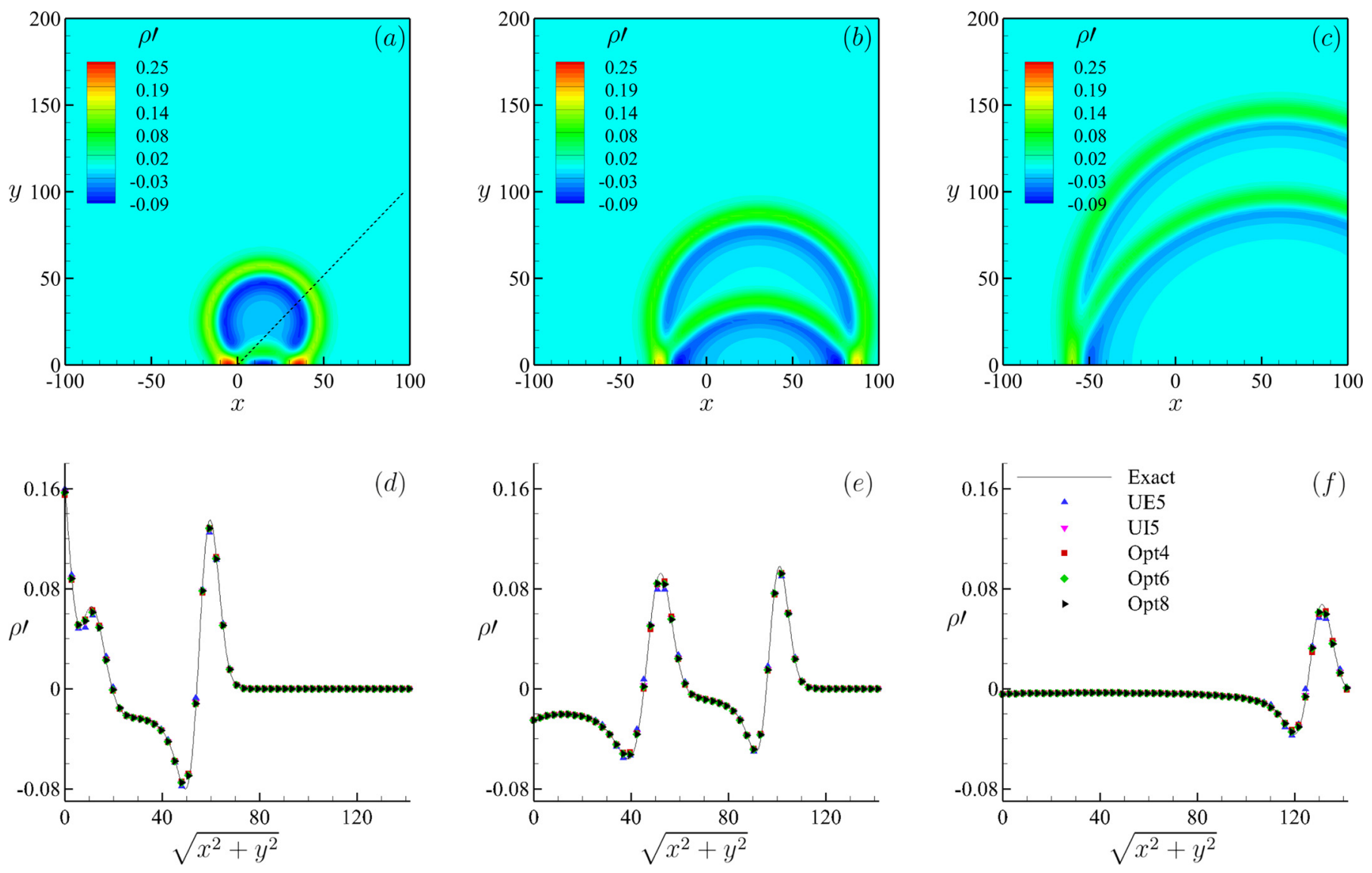

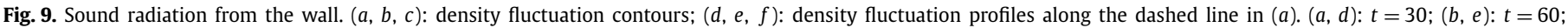
$(c, f): t=120$

half of the computational domain is considered. The grids for the two and three-cylinder cases are spaced with $\Delta x=\Delta y=0.035$ and $\Delta x=\Delta y=0.035$ respectively. A periodic acoustic source is introduced by adding

$$
S=\varepsilon \exp \left[-25(\ln 2)\left(x^{2}+y^{2}\right)\right] \sin (8 \pi t)
$$

to the energy equation in Eq. (7). $\varepsilon=0.001$. Fig. 10 show both the instantaneous and root-mean-square (r.m.s.) of pressure fluctuation contours. The waves radiated from the cylinders are clearly captured. Fig. 11 compares the r.m.s. pressure fluctuations on the cylinder surfaces with the analytical solutions. It is observed that UI5 shows a dramatic improvement over UE5 and the optimized schemes (Opt4, Opt6 and Opt8) give additional improvements over UI5.

\subsection{Turbulence flow of periodic hill}

Flow over periodic hill is carried out to validate the simulation of turbulence with DDES in separated flows. This case is welldocumented by experiment [14] and numerical simulations [38][39][40]. Following the compressible settings by Ziefle et al. [40], the Reynolds number based on the hill height $h$ and the bulk velocity $U_{b}$ is 10595 where $U_{b}$ is defined by the velocity above the hill crest. The Mach number is 0.27 based on the $U_{b}$ and the sound speed at wall. The computational domain is $[0,9 h] \times[0,3.035 h] \times[0,4.5 h]$ consisting of $196 \times 128 \times 186$ cells. Periodic boundary conditions are imposed in the streamwise and spanwsie directions. Isothermal non-slip wall condition is imposed on the top and bottom walls. Fig. 12 depict the main features of the statistic quantities at five stations along $x$-axis direction, which are accumulated over 30 flow-through times after 30 flow-through times for flow developing. Good consistence with the reference data is observed.

\subsection{Tonal noise radiated from the 2D NACA0012 airfoil}

A laminar flow past a NACA0012 airfoil at $5^{\circ}$ angle of attack [22] is investigated as the validation of our implementation of FW- $\mathrm{H}$ acoustic analogy formulation. The Reynolds number based on the airfoil chord $c$ is 5000 and the Mach number is 0.3 . The observer points are located on a circle at a distance with radius of 17c. Fig. 13 (a) shows an obviously periodic vortex shedding street from the trailing edge and $(b)$ draws the far-field directivity pattern where the convective effect has a noticeable influence. The small discrepancy between the DNS data [22] and the present results may stem from the neglect of the volume integrations. 

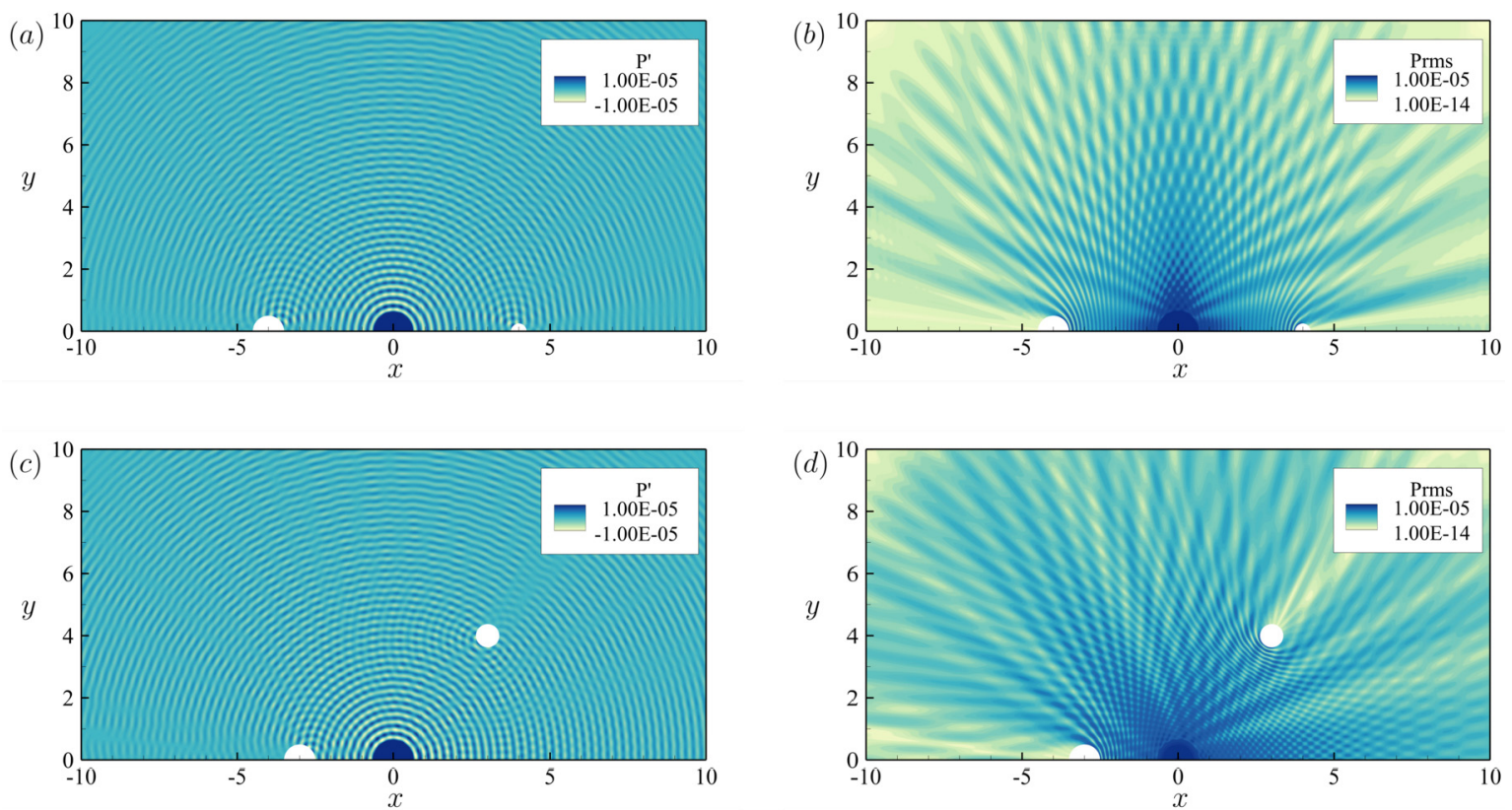

Fig. 10. Acoustic field. $(a, c)$ : the snapshot of acoustic pressure field. $(b, d)$ : r.m.s. acoustic pressure field. $(a, b)$ : two cylinders; $(c, d)$ : three cylinders.
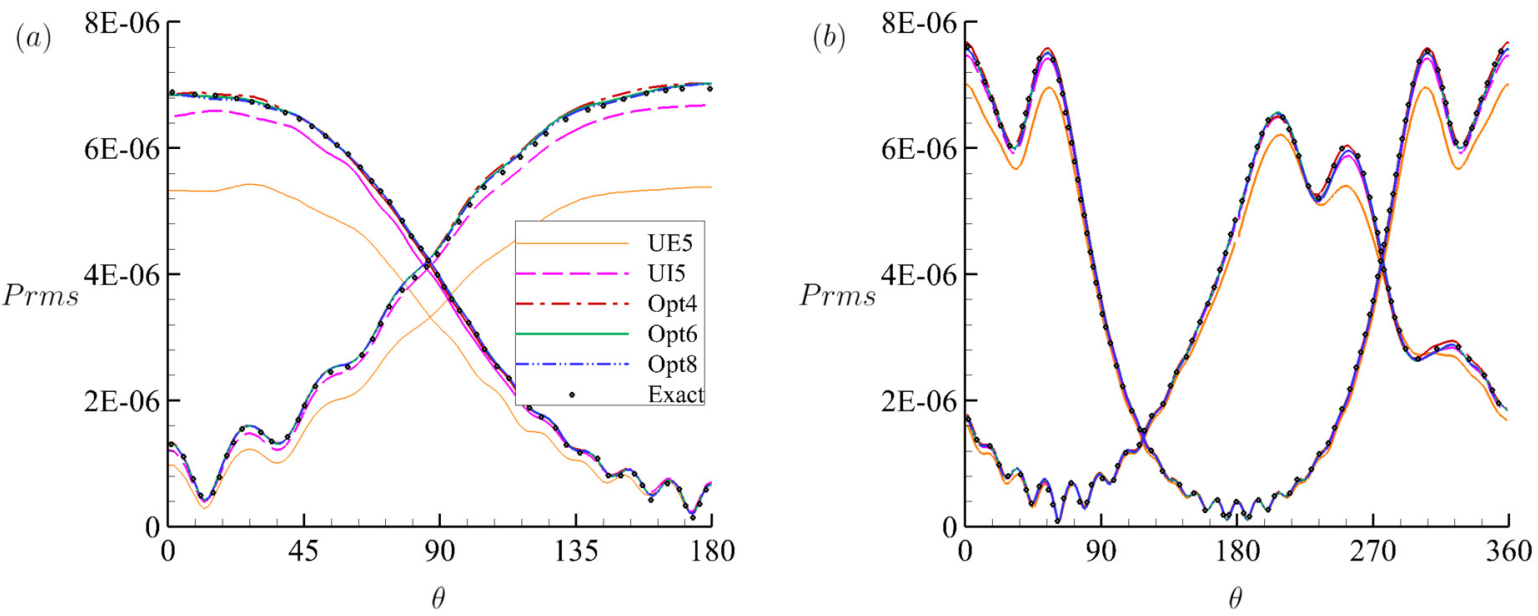

Fig. 11. R.m.s. pressure fluctuations on the surface of cylinders. (a): two cylinders; $(b)$ : triple cylinders. The legend for $(b)$ is same with that in (a).

\section{Radiation noise of rod-airfoil configuration}

The radiated noise by the wake-airfoil interaction is investigated through a rod-airfoil configuration [24]. The rod with diameter $d=$ $0.01 \mathrm{~m}$ is placed upstream of the symmetric NACA 0012 airfoil with chord $c=0.1 \mathrm{~m}$. The distance between the rod and the airfoil is $1 c$. The incoming Mach number is given by $M a=0.2$. The Reynolds number based on the rod diameter and the airfoil chord are $R e_{d}=4.8 \times 10^{4}$ and $R e_{c}=4.8 \times 10^{5}$ respectively. Some of the previous numerical studies are summarized in Table 3.

\subsection{Computational setup and cost}

The computational domain extends from $-8 c$ to $16 c$ in the streamwise direction and from $-8 c$ to $8 c$ in the crosswise direction. A $2 \mathrm{D}$ mesh is initially generated on $x-z$ planer and then extruded along the $y$-axis for $0.3 c$ to yield the 3D grid. Details regarding the grid topology are shown in Fig. 14. No-slip wall boundaries are applied on the solid surfaces and periodic condition is applied on the spanwise direction.

Three set of grids are carried out for mesh sensitivity study, namely Grid A, Grid B and Grid C, which consist of 3-million, 7-million and 15.8-million cells respectively, with 28 cells, 44 cells and 60 cells in spanwise direction respectively. On the circumferential direction of the rod surface, 176 cells, 200 cells and 216 cells are distributed corresponding to Grid A, Grid B and Grid C. On the airfoil surface, 344 cells, 400 cells and 488 cells are distributed respectively. The mean scaled $\Delta z^{+}$of the first cell in the wall-normal direction is reported to be below 1 for all grids. The estimated spanwise $\Delta y^{+}$is reported to be 264 for Grid A, 127 for Grid B and 93 for Grid C.

The implicit dual time-stepping method is adopted. The physical time interval is chosen as 0.02 and 20 pseudo steps are given per real time step. 16384 real time units in total (corresponding to physically $0.09 \mathrm{~s}$ or 64 periods through the airfoil) are collected for flow statistical quantities. In order to obtain the power spectral density (PSD) estimations, these data are split into 7 segments with an 

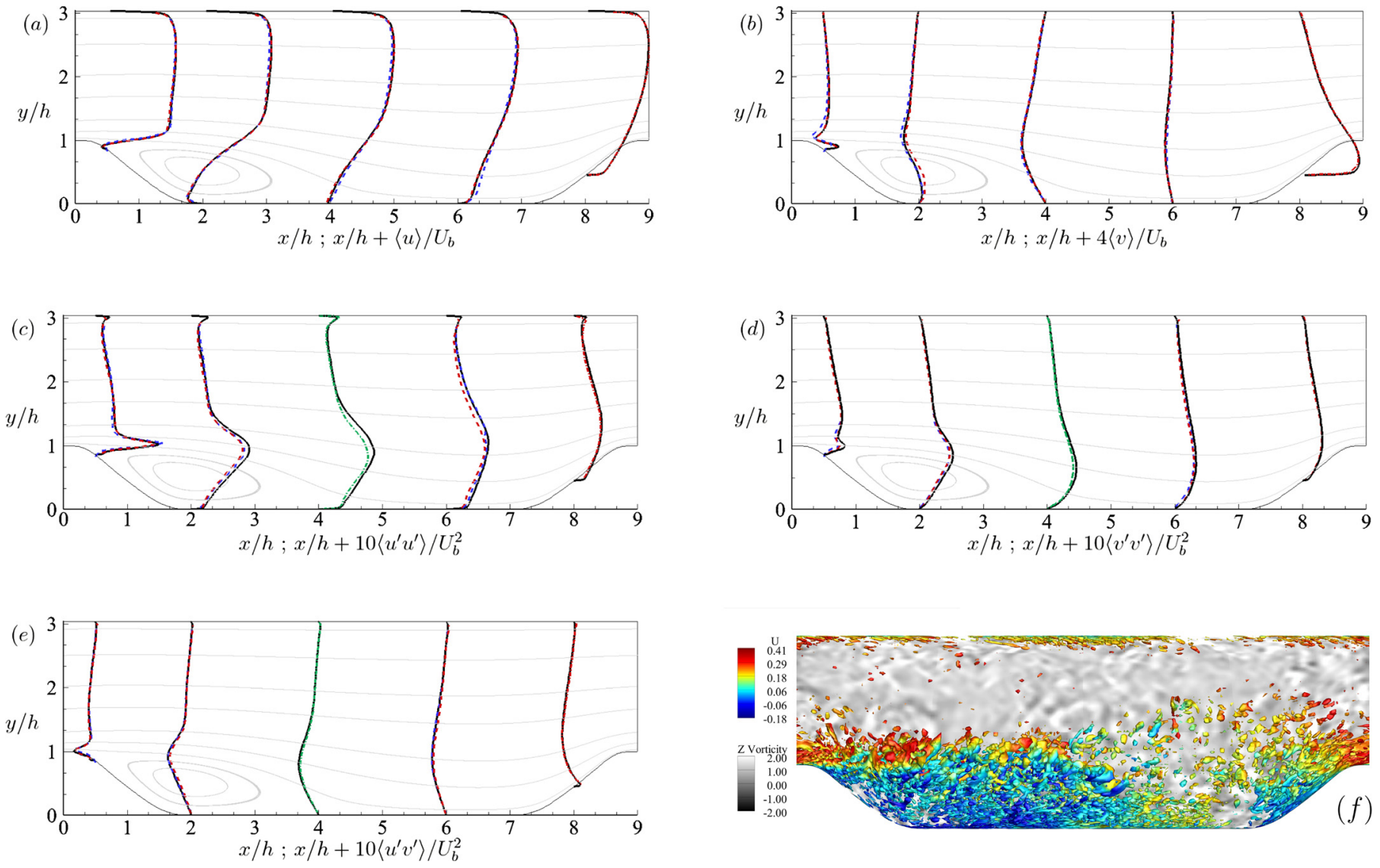

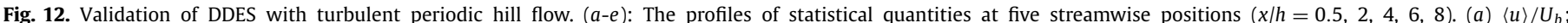

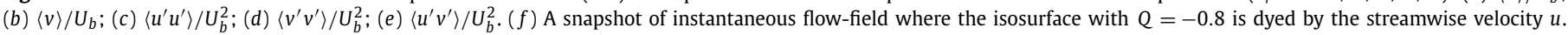

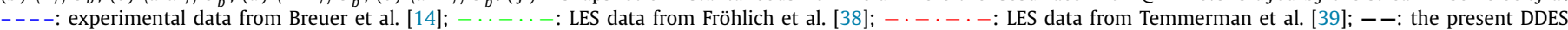
results.
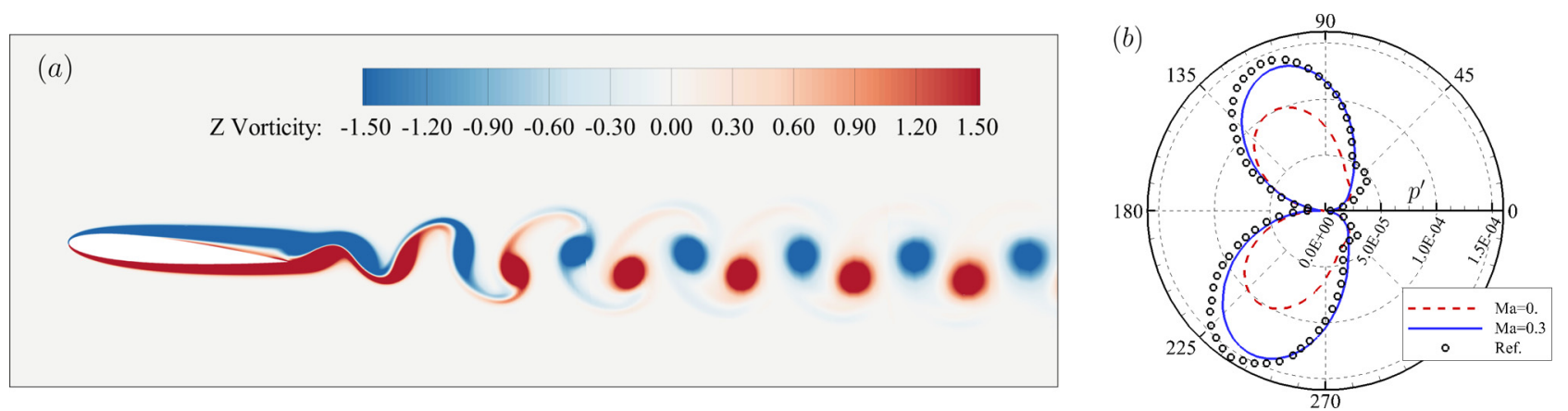

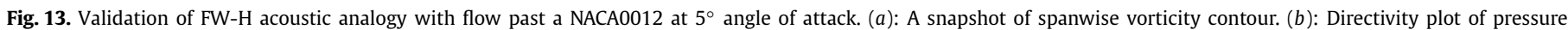
fluctuation $p^{\prime}$ at $17 c$ distance from NACA0012. $p^{\prime}$ is non-dimensional by the freestream density and sound of speed.

overlapping of $50 \%$ to eliminate the variance. Case 3 was conducted using 80 cores for 18 days on a workstation with Intel(R) Xeon(R) Platinum 8163 CPU @ 2.50 GHz.

\subsection{Near-field aerodynamic results}

Fig. 15 visualizes an overview of the instantaneous flow-field, where the flow separation takes place initially on the surface of rod and then subsequently transits in the shear layer before finally forming the unsteady three dimensional vortex shedding street. The turbulence structures further move downstream and then impact on the leading edge of the airfoil. The Karman vortex shedding wake behind the rod and the wake-airfoil interaction are two major components for the acoustics.

Fig. 16 show histories of lift and drag coefficients on the rod and airfoil surfaces and their PSD profiles. $\mathrm{Cl}$ and $\mathrm{Cd}$ are given by $\mathrm{Cl}=$ $2 \cdot$ lift $/\left(\rho_{\infty} U_{\infty}^{2} A\right)$ and $C d=2 \cdot \operatorname{drag} /\left(\rho_{\infty} U_{\infty}^{2} A\right)$ where the reference area $A$ for rod and airfoil are $A_{\text {rod }}=0.3 c \cdot 0.1 c$ and $A_{\text {airfoil }}=0.3 c \cdot c$ respectively. Fig. $16(b)$ shows that the vortex shedding frequency at $f_{0}=0.193$, corresponding to the experimental $f_{0}=0.19$ by Jacob et al. [25], and its second harmonic $3 f_{0}=0.58$ are precisely predicted by the lift coefficients. And the airfoil oscillates at the exactly same frequency with the rod. The drag coefficients exhibit the first harmonic peak at $2 f_{0}=0.386$. 
Table 3

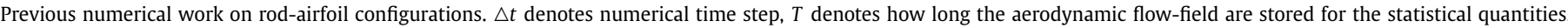

\begin{tabular}{|c|c|c|c|c|c|c|c|c|}
\hline Authors & Year & Mesh & Method & Cells $\left(\times 10^{6}\right)$ & Span length & Span cells & $\Delta t(s)$ & $T(s)$ \\
\hline Casolino et al. [41] & 2003 & 2D Structured & Compressible URANS & 0.05464 & - & 1 & $6 \times 10^{-8}$ & $3.15 \times 10^{-2}$ \\
\hline Boudet et al. [42] & 2005 & 3D Structured & Compressible LES & 2.4 & $0.3 c$ & 30 & $3 \times 10^{-8}$ & - \\
\hline Peth et al. [43] & 2006 & 3D Structured & Incompressible LES & 3.14 & $0.3 c$ & 30 & - & $3.6 \times 10^{-2}$ \\
\hline Berland et al. [44] & 2010 & 3D Chimera & Compressible LES & 20 & $0.3 c$ & 44 & $6.5 \times 10^{-8}$ & $6.5 \times 10^{-2}$ \\
\hline Galdéano et al. [45] & 2010 & 3D Unstructured & Compressible DES & $\begin{array}{l}3.5 \\
10 \\
15\end{array}$ & $\begin{array}{l}0.3 c \\
3 c \\
4.5 c\end{array}$ & - & $2 \times 10^{-5}$ & $1.2 \times 10^{-1}$ \\
\hline Eltaweel et al. [46] & 2011 & 3D Unstructured & Incompressible LES & 22.3 & $0.314 c$ & 90 & - & $6.7 \times 10^{-2}$ \\
\hline Giret et al. [47] & 2012 & 3D Unstructured & Compressible LES & $\begin{array}{l}4.25 \\
24.2\end{array}$ & $\begin{array}{l}0.35 c \\
0.7 c\end{array}$ & 59 & $2.5 \times 10^{-7}$ & $1.5 \times 10^{-1}$ \\
\hline Shell et al. [48] & 2013 & 3D Patched & Compressible DES & 95 & $\begin{array}{l}1 c \\
3 c\end{array}$ & - & $1 \times 10^{-5}$ & $2 \times 10^{-1}$ \\
\hline Agrawal et al. [49] & 2014 & 3D Structured & $\begin{array}{l}\text { Compressible \& } \\
\text { Incompressible LES }\end{array}$ & 19 & $1 c$ & - & $4 \times 10^{-5}$ & $1.7 \times 10^{-2}$ \\
\hline Giret et al. [50] & 2015 & 3D Unstructured & Compressible LES & $\begin{array}{l}31.5 \\
42.9 \\
89.5\end{array}$ & $0.35 c$ & - & $\begin{array}{l}1.5 \times 10^{-7} \\
5 \times 10^{-8} \\
2 \times 10^{-8}\end{array}$ & $2 \times 10^{-2}$ \\
\hline Jiang et al. [51] & 2015 & 3D Structured & Compressible ILES & 16 & $0.3 c$ & 44 & $1.389 \times 10^{-6}$ & $2.78 \times 10^{-2}$ \\
\hline Agrawal et al. [52] & 2016 & 3D Structured & Incompressible LES & $\begin{array}{l}10 \\
19 \\
64\end{array}$ & $0.3 c$ & 80 & - & $2.9 \times 10^{-2}$ \\
\hline Zhou et al. [53] & 2017 & 3D Unstructured & Compressible DDES & 6 & $0.35 c$ & 64 & $1 \times 10^{-6}$ & $3.47 \times 10^{-2}$ \\
\hline Tong et al. [54] & 2017 & 3D Structured & Compressible LES & $\begin{array}{l}3.46 \\
5.15\end{array}$ & $0.2 c$ & 32 & $\begin{array}{l}1 \times 10^{-5} \\
2 \times 10^{-6}\end{array}$ & $2.2 \times 10^{-1}$ \\
\hline Leveque et al. [55] & 2017 & 3D Unstructured & Compressible WMLES & 20 & $0.35 c$ & - & $\begin{array}{l}1.68 \times 10^{-7} \\
5.38 \times 10^{-6}\end{array}$ & $2.5 \times 10^{-1}$ \\
\hline Chen et al. [56] & 2018 & 3D Structured & Incompressible LES & 3.47 & $0.2 c$ & 32 & $2 \times 10^{-6}$ & $4 \times 10^{-2}$ \\
\hline Sharma et al. [57] & 2019 & 3D Unstructured & Compressible DES & 4 & $0.4 c$ & - & $1 \times 10^{-5}$ & $1 \times 10^{-1}$ \\
\hline $\begin{array}{l}\text { Present Case } 1 \\
\text { Present Case } 2 \\
\text { Present Case } 3\end{array}$ & - & 3D Structured & $\begin{array}{l}\text { Compressible } \\
\text { DDES }\end{array}$ & $\begin{array}{l}3(\text { Grid A) } \\
7(\text { Grid B) } \\
15.8(\text { Grid C) }\end{array}$ & $0.3 c$ & $\begin{array}{l}28 \\
44 \\
60\end{array}$ & $5.5 \times 10^{-6}$ & $9 \times 10^{-2}$ \\
\hline
\end{tabular}
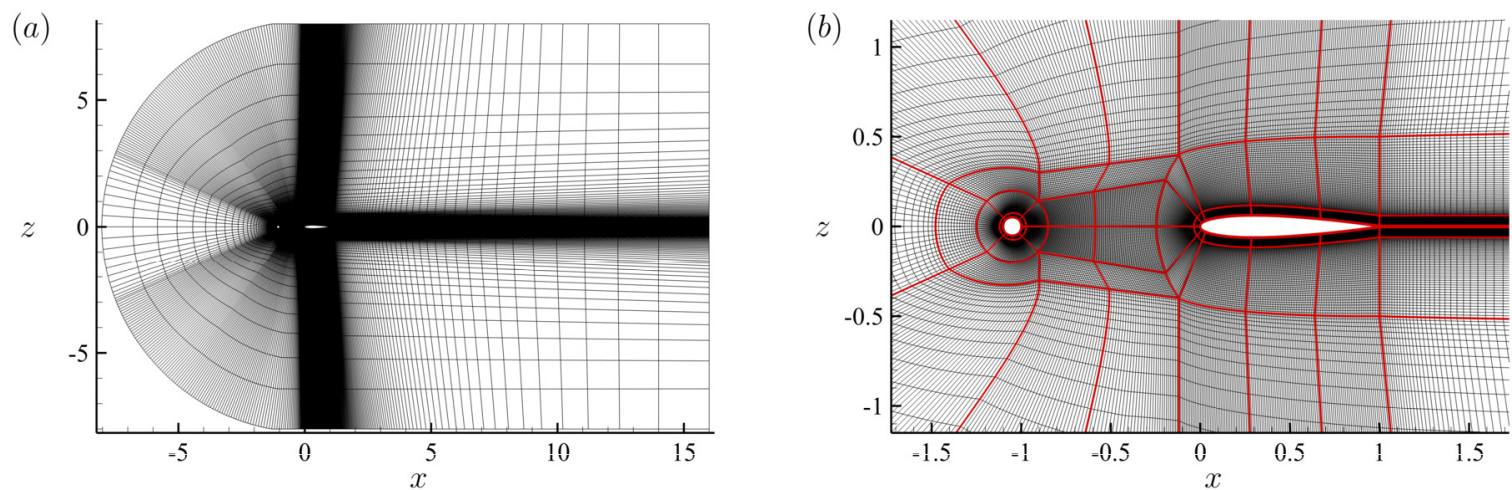

Fig. 14. (a): Cross-section view of the computational domain; (b): Grid topology in the vicinity of geometry.

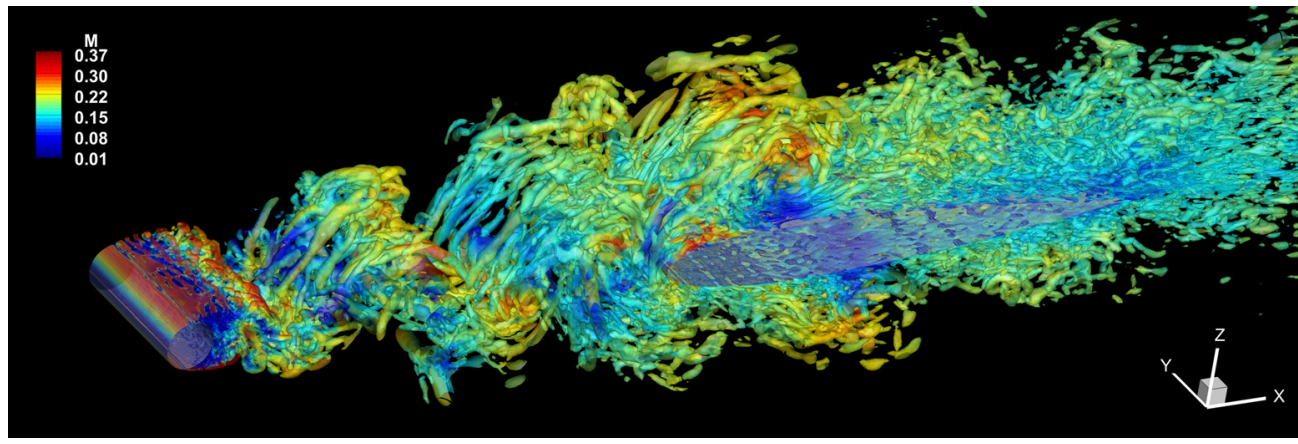

Fig. 15. A snapshot of the instantaneous flow-field where the isosurface of $Q=2$ is dyed by Mach number 

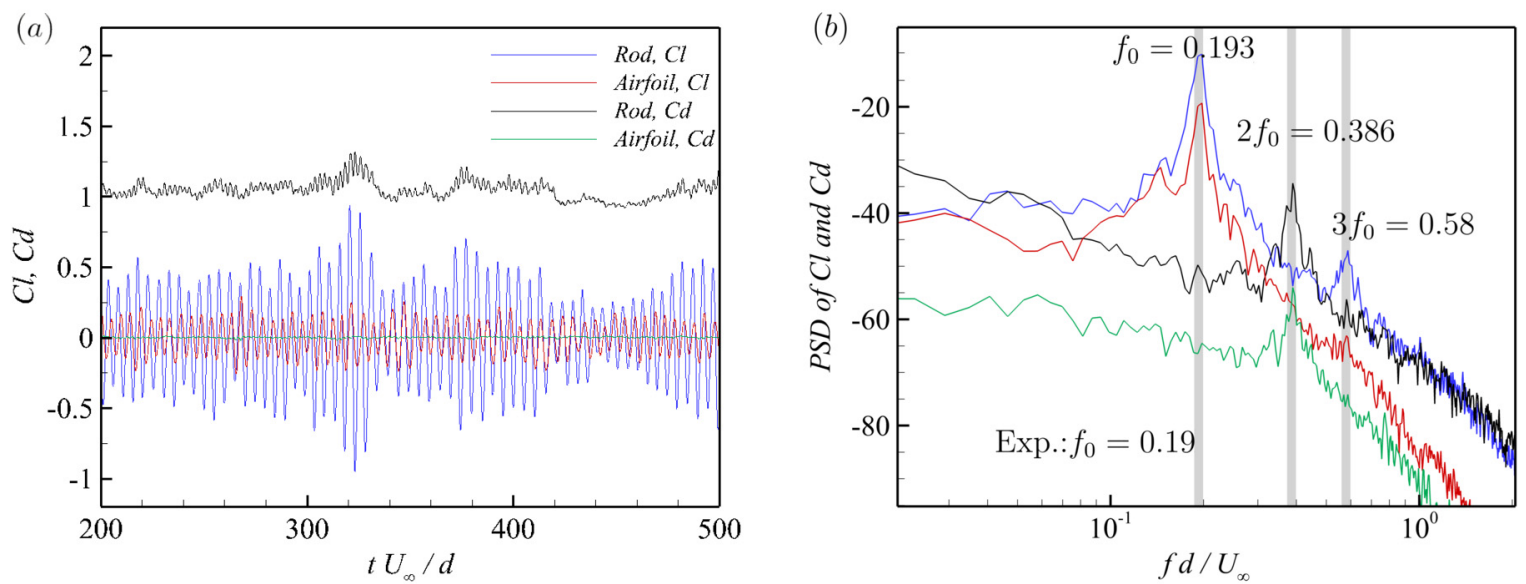

Fig. 16. Unsteady lift and drag coefficients on the surface of rod and airfoil. (a): Time history of $C l$ and $C d$; (b): PSD of $C l$ and $C d$.
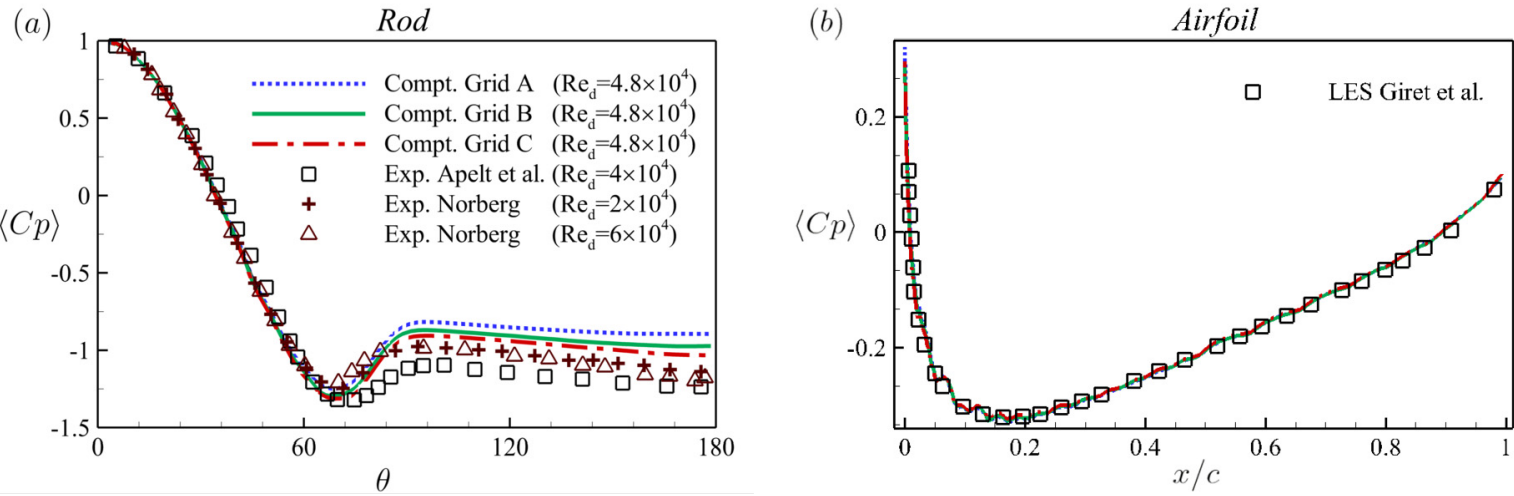

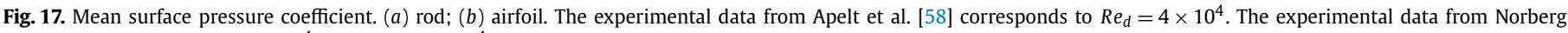
[59] corresponds to $R e_{d}=2 \times 10^{4}$ and $R e_{d}=6 \times 10^{4}$.

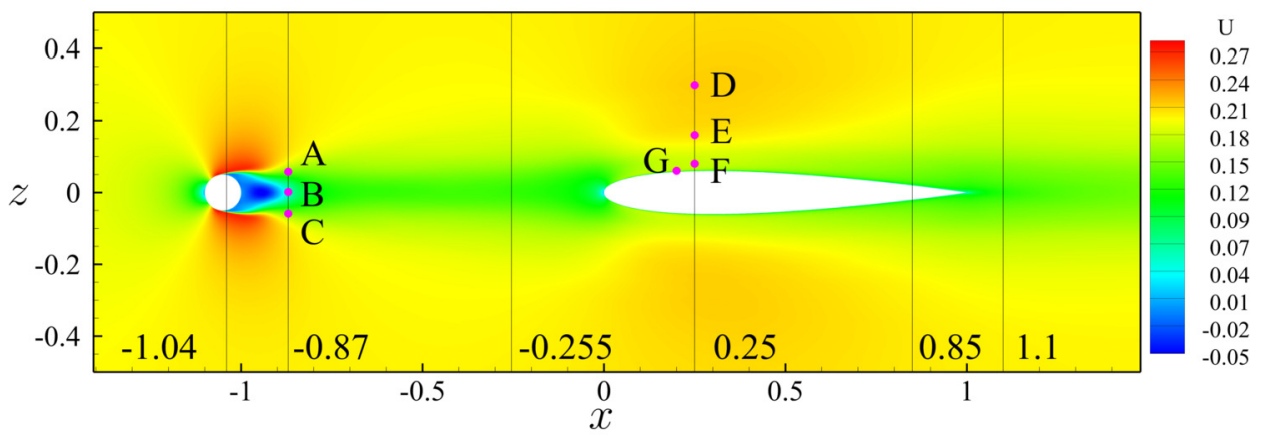

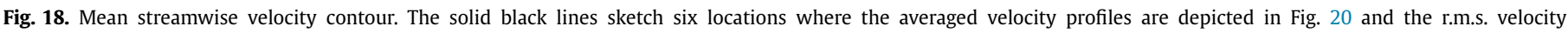

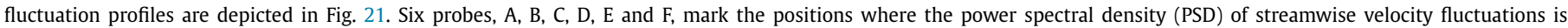
investigated in Fig. 22. Additional probe G denotes the place where PSD of pressure fluctuation is shown in Fig. 23.

The distributions of time-averaged pressure coefficient $\langle C p\rangle=2 \cdot\left(\langle p\rangle-p_{\infty}\right) /\left(\rho_{\infty} U_{\infty}^{2}\right)$ on the surface of the rod and airfoil are shown in Fig. 17. Due to lack of experimental pressure coefficient at $R e_{d}=4.8 \times 10^{4}$, the measured data for an isolated cylinder with closer Reynolds numbers by Apelt and West [58] and Norberg [59] are used for reference in Fig. 17 (a). For the airfoil, the LES data by Giert et al. [50] are taken for reference in Fig. $17(b)$. The grid sensitivity test indicates that the result yielded on Grid $C$ is more agreeable with the measured data than that on Grid A and Grid B. Yet, the pressure for $100^{\circ}<\theta<180^{\circ}$ is slightly over-predicted. This is likely because the resolution of Grid $C$ on the spanwise direction for rod is still insufficient. The estimated spanwise $\Delta y^{+}=93$ for Grid $C$ is still too large and may degrade the results' accuracy even with a wall-normal $\Delta z^{+}$of 1 . Similar comment is also presented by Giret et al. [50]. Another concern for this is the experiments of Apelt and West [58] and Norberg [59] which are conducted for an isolated cylinder at other Reynolds numbers. In addition, as is pointed out by Jiang et al. [51], the airfoil behind the rod may cause pressure increase for $\theta>90^{\circ}$ in Fig. 17 (a).

Fig. 18 shows the contour of mean streamwise velocity with six $x$-axis locations and seven probes for further analysis. Fig. 19 is depicted to show the mean and r.m.s. velocity profiles on the wake centerline. They are compared with the LES data from Giret et al. 

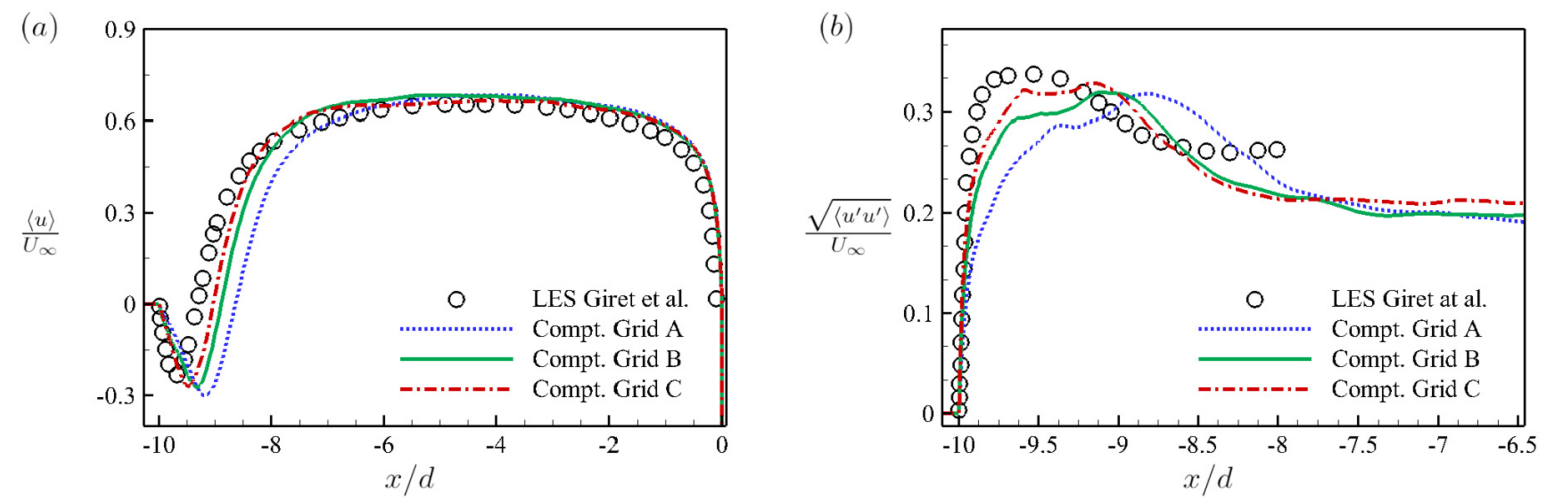

Fig. 19. Mean and r.m.s. streamwise velocities along the $x$-axis. (a): mean streamwise velocity; $(b)$ : r.m.s. streamwise velocity.
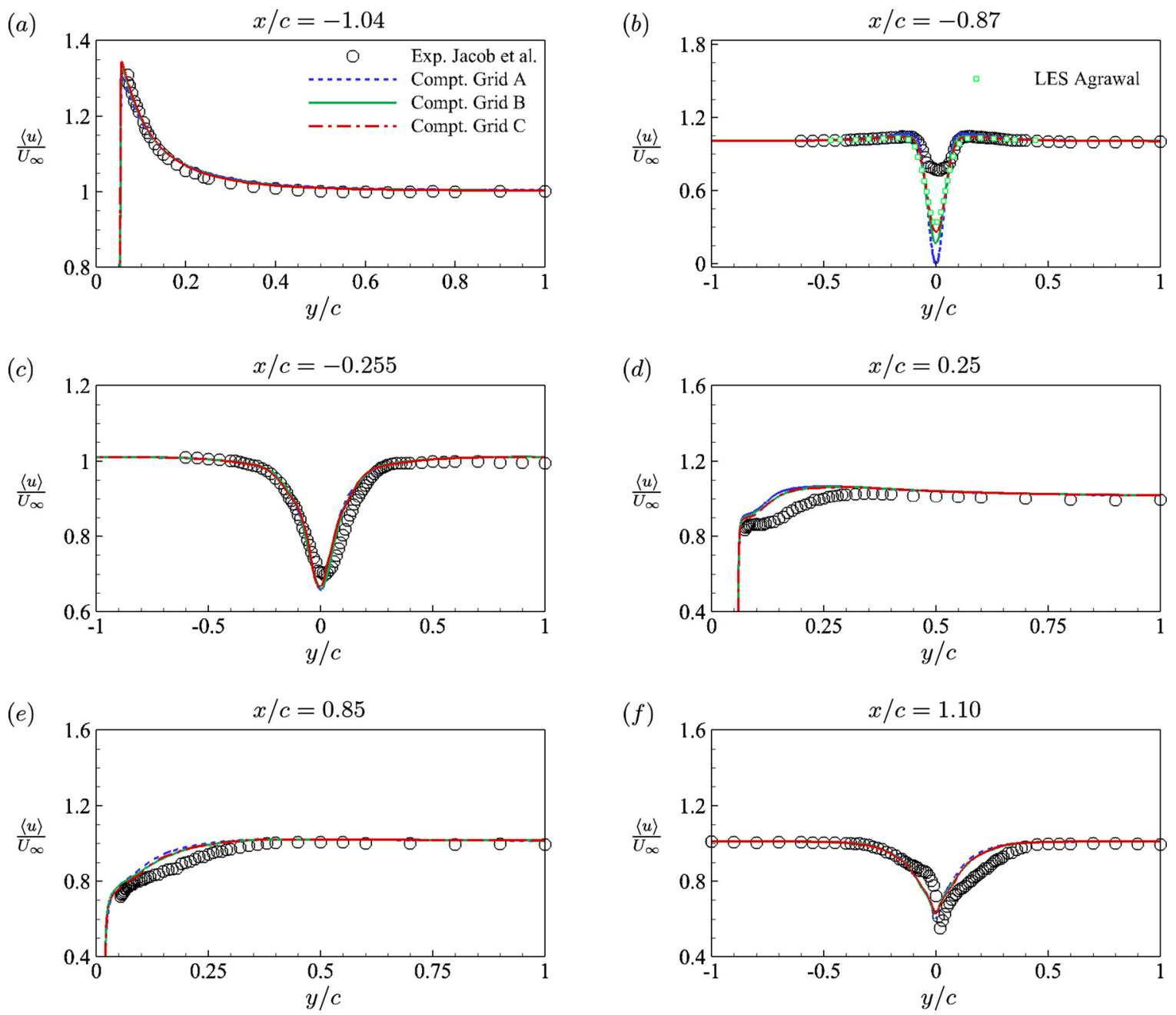

Fig. 20. Mean streamwise velocity profiles at six $x$-axis positions. Experimental data by Jacob et al. [24] and Agrawal's LES data [60] are for comparison.

[50]. In Fig. 19 (a), the location of minimum streamwise velocity approaches the LES result with grid refinement. And in Fig. 19 (b), the location where $\sqrt{\left\langle u^{\prime} u^{\prime}\right\rangle} / U_{\infty}$ reach its maximum gets closer to the LES data as the grid resolution increases.

The averaged streamwise velocity $\langle u\rangle / U_{\infty}$ profiles and r.m.s. streamwise velocity fluctuation $\sqrt{\left\langle u^{\prime} u^{\prime}\right\rangle} / U_{\infty}$ profiles at six $x$-axis locations are compared with the experimental data in Fig. 20 and Fig. 21 respectively. Noticeably, except for the results at $x / c=-0.87$, the numerical solutions agree well with the experimental data. But, our results at $x / c=-0.87$ agree well with that by Agrawal and Sharma [49][60]. They describe concerns about the measured data due to it that the peak velocity deficit in the wake is expected to reduce with the distance away from the rod, which indicates the velocity deficit in Fig. 20 (b) should be more than that in Fig. 20 (c). The measured data at $x / c=1.1$ behaves with a slight asymmetric pattern. And this asymmetry has been clarified by Jacob et al. [24] that the rod in the experiment is slightly misaligned with the airfoil by about $2 \mathrm{~mm}$ in the vertical direction, resulting in a $2^{\circ}$ angle of attack of the airfoil. 

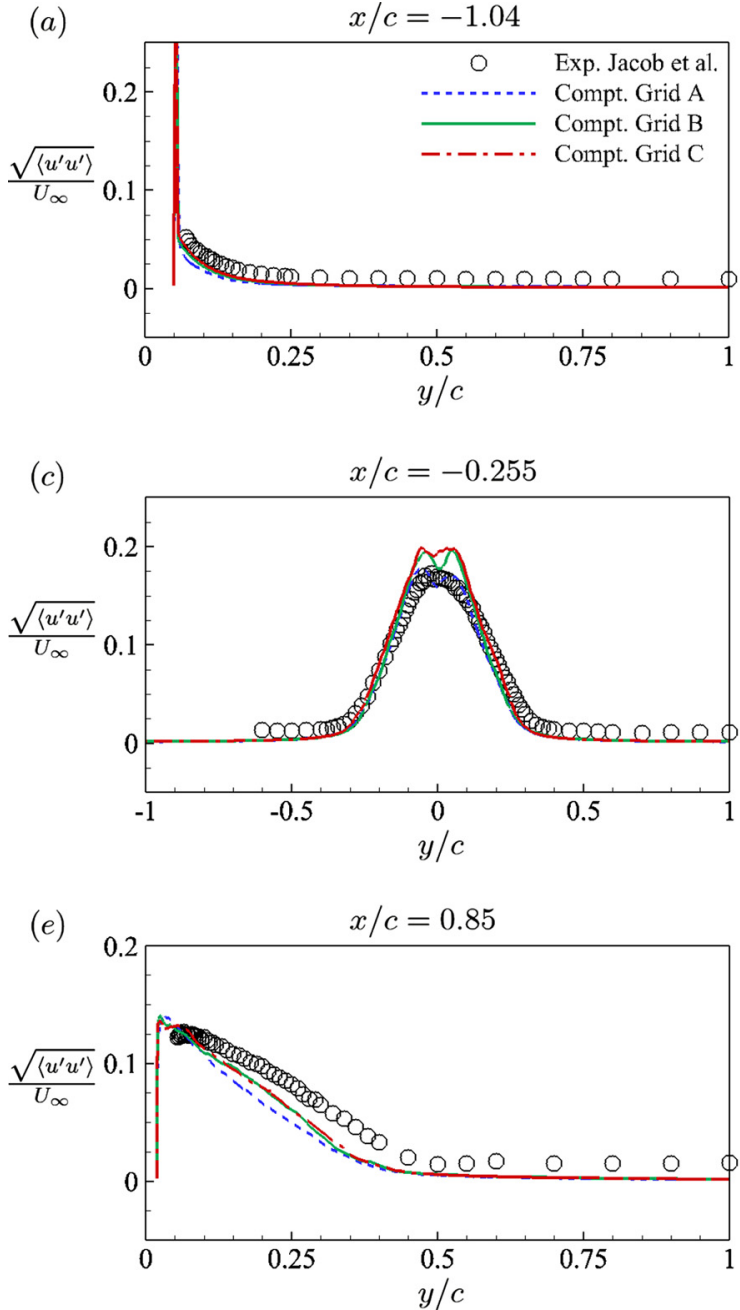
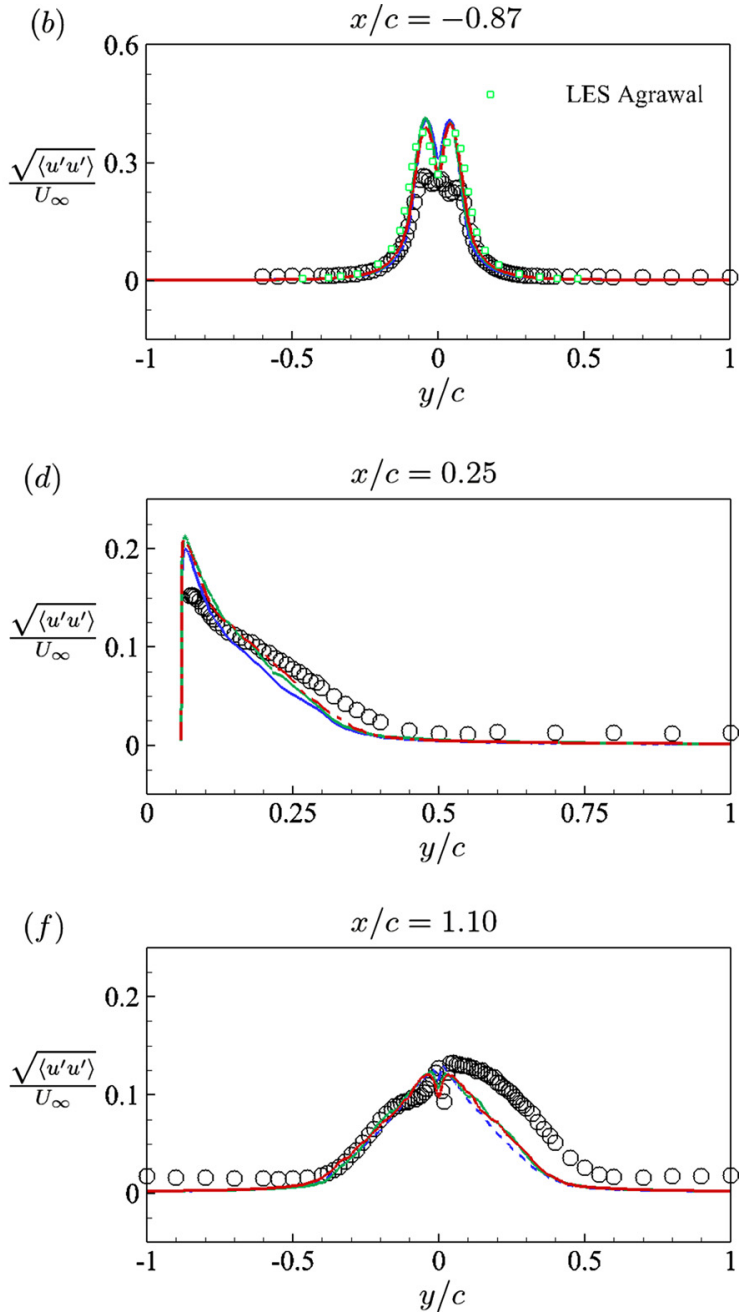

Fig. 21. R.m.s. streamwise velocity fluctuation profiles at six $x$-axis positions. Experimental data by Jacob et al. [24] and Agrawal's LES data [60] are for comparison.

The power spectral density (PSD) of streamwise velocity fluctuations at six locations are shown in Fig. 22 where probe A, B and C are in the near wake of $\operatorname{rod}(x / c=-0.87)$ and $\mathrm{D}, \mathrm{E}$ and $\mathrm{F}$ are above the airfoil $(x / c=0.25)$. Overall, both the fundamental peak and broadband components satisfactorily agree with the measured data [25]. For probe A and C, a fundamental peak at $f_{0}=0.193$ due to vortex shedding as well as its harmonic peaks at $2 f_{0}$ and $3 f_{0}$ is resolved. For probe $\mathrm{B}$, only a minor peak at the first harmonic $2 f_{0}$ is observed whereas the main shedding peak disappears. This is because probe $B$ is influenced by the vortices shedding from the upside and downside of the rod equally. In terms of probes $\mathrm{D}, \mathrm{E}$ and $\mathrm{F}$, the visible peaks occurred at fundamental frequency indicate that the vortices shed from the rod still dominate the velocity field surrounding the airfoil. For the nearest point $\mathrm{F}$, the calculated data agrees well with the experimental data. Yet, the prediction at point $\mathrm{D}$ is lower than the measured data. The reason may be due to the insufficient grid resolution. A similar result is also obtained by Shell [48].

Fig. 23 shows the PSD of pressure fluctuations at location G on the airfoil surface. A relatively good agreement with the experiment by Jacob et al. [25] is observed. Yet, nearly $4 \mathrm{~dB}$ is over-predicted for the broadband range. This discrepancy is also noticed by Giret et al. [50]. Their data by LES technique on the finest mesh (containing $89.5 \times 10^{6}$ cells) is also included. They concerns the discrepancy may be related to an insufficient spanwise length or the curvature discontinuity at the airfoil leading edge.

\subsection{Far-field acoustic results}

For low Mach number flows, the omission of quadrupole source has little influence on the noise radiation [17]. Besides, as mentioned in Table 3, the spanwise length in CFD $(0.3 c)$ is typically a small portion of that in experiment ( $3 c)$. Hence, the input data for FW-H equation are replicated 10 times in the spanwise direction. Fig. 24 (a) shows that the observers are placed at a distance of $1.85 \mathrm{~m}$ (corresponding to $18.5 c)$ from the airfoil center. In Fig. $24(b-d)$, the PSD of pressure fluctuations at three far-field locations $\left(\theta=60^{\circ}, 90^{\circ}\right.$ and $\left.120^{\circ}\right)$ is observed to agree with the experimental data well. Fig. 24 (e) indicates the dominant frequency for the observer at $180^{\circ}$ is twice of that for the observers at $\theta=60^{\circ}, 90^{\circ}$ and $120^{\circ}$. This is because along the horizontal direction, the lift dipole is absent and the drag dipole dominates. The broadband contents at $180^{\circ}$ also exhibit lower intensities. Fig. $24(f)$ gives the far-field directivity plot which resembles a dipole in the normal direction and is also in good agreement with the experimental data. 

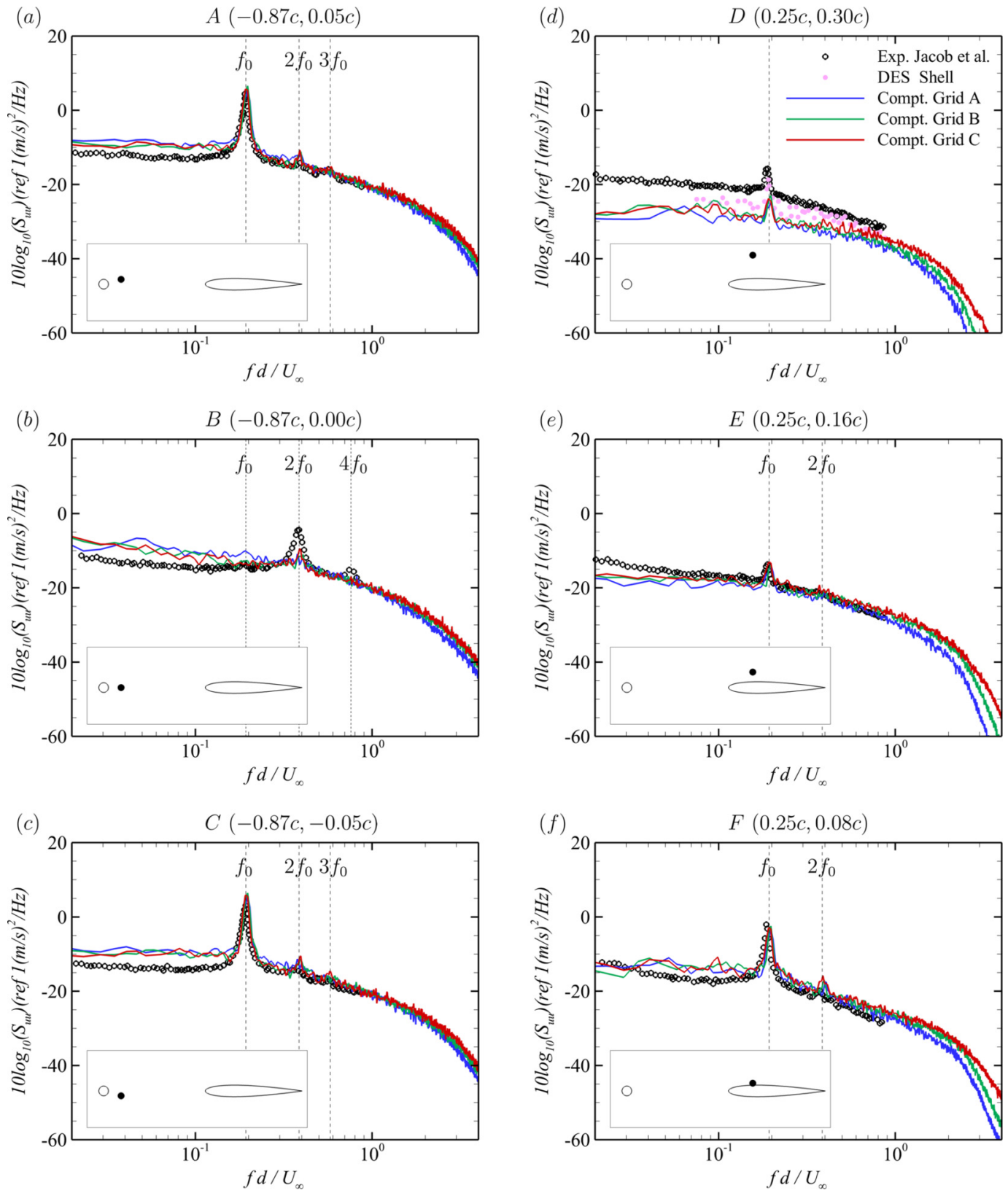

Fig. 22. PSD of streamwise velocity fluctuations at six probes in the flow-field. $(a-c)$ in the near wake of the rod; ( $d$ - $f$ ) in the upper side of the airfoil.

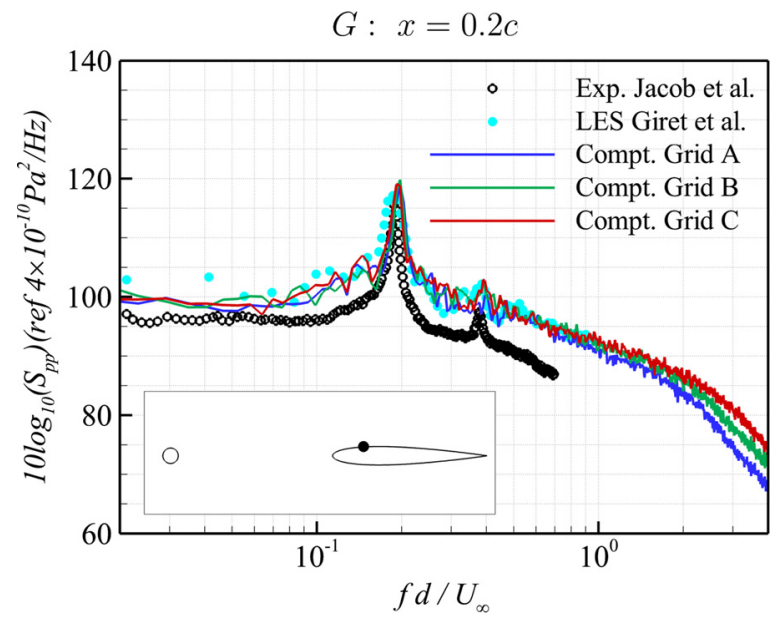

Fig. 23. PSD of pressure fluctuations at location $\mathrm{G}$, where $x / c=0.2$, on the airfoil. 
(a)
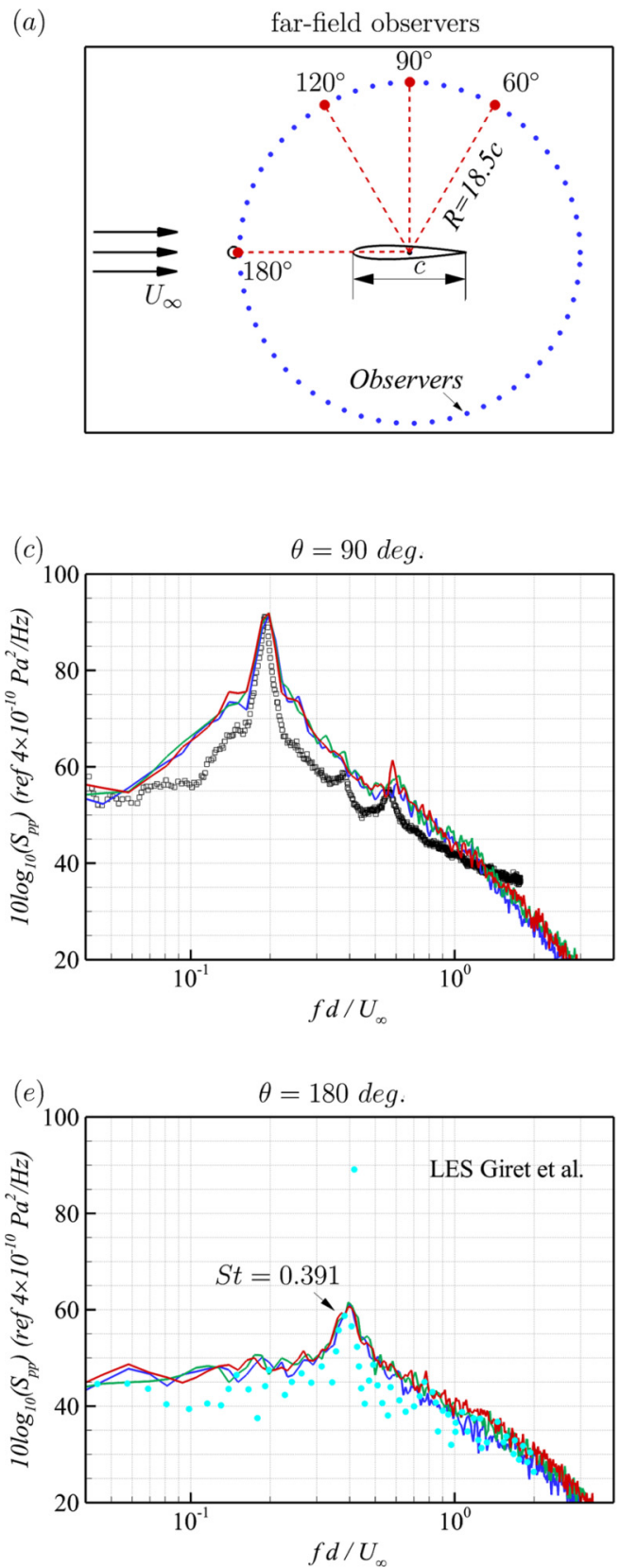
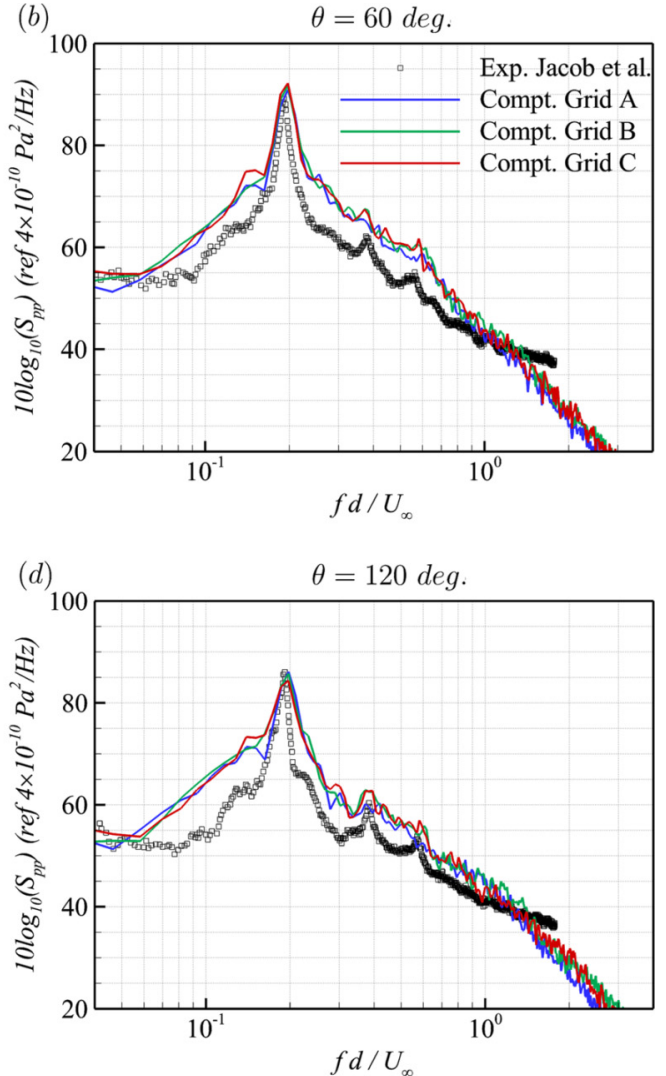

$(f)$

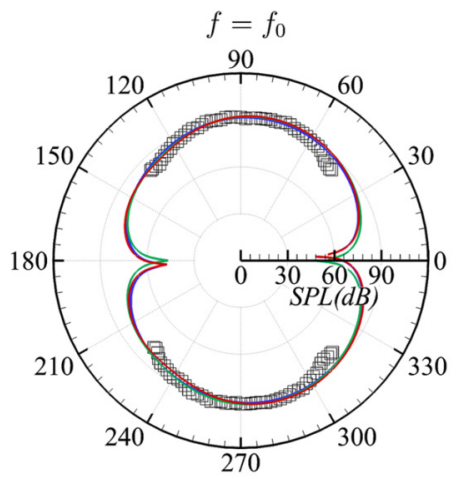

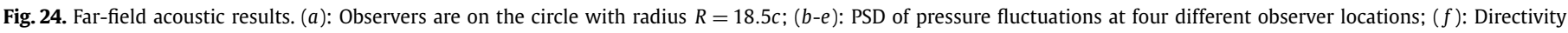

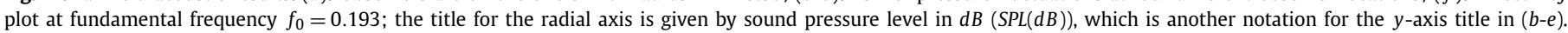

Fig. 25 is further drawn to analyze the relative contribution of rod and airfoil to the far-field acoustics. In Fig. 25 (a), the predominance of airfoil for the low-frequencies indicates the major contributor for the acoustics is the wake-airfoil interaction. In terms of the highfrequencies, the rod and airfoil contributes almost equally to the observer at $\theta=90^{\circ}$. Fig. $25(b-d)$ give the directivity plots at three frequencies $f_{0}=0.193,2 f_{0}=0.386$ and $3 f_{0}=0.58$, which correspond to the fundamental frequency, first harmonic and second harmonic respectively.

\section{Conclusions}

This paper focuses on the radiation noise of a rod-airfoil configuration based on a high-order cell-centered finite difference method (CCFDM) and Ffowcs Williams-Hawkings (FW-H) acoustic analogy.

First of all, to achieve low-dissipation and low-dispersion properties for aeroacoustics, three optimized compact schemes (Opt4, Opt6 and Opt8) for CCFDM are proposed. The expected accuracy (4th-, 6th- and 8th-order respectively) are validated by the isentropic vortex problem on both uniform and wavy grids. The superior spectral properties are also demonstrated by the benchmark cases from computational aeroacoustics workshops. Secondly, for massively separated turbulence flows, DDES is adopted and validated with a canonical periodic hill problem. For the far-field radiated acoustics, the FW-H acoustic analogy is used, which is validated by a laminar flow past a NACA0012 airfoil. 
$(a)$

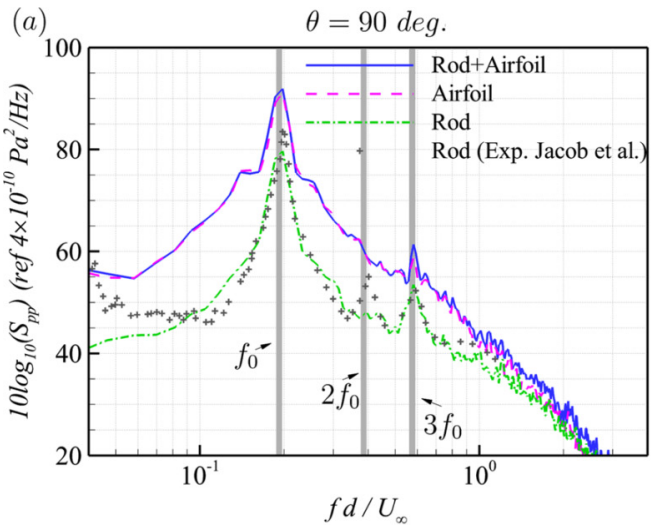

(c) $(b)$

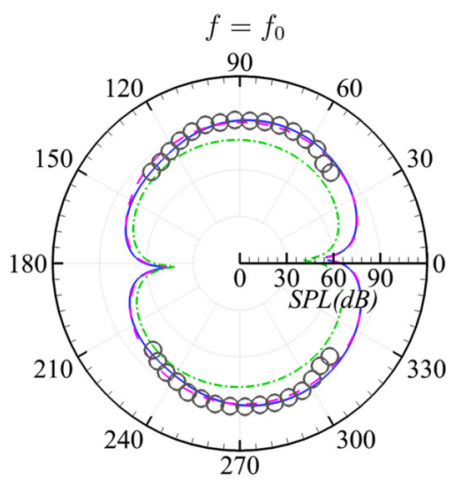

$(d)$

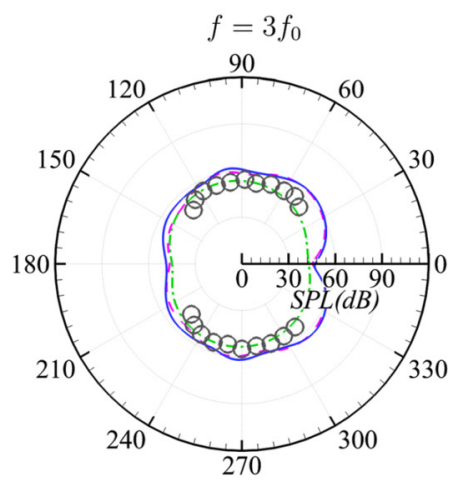

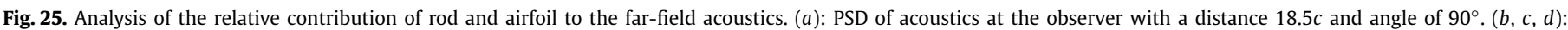

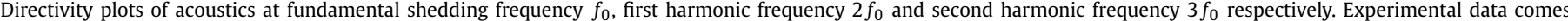
from Jacob et al. [24].

Finally, the radiated noise of the rod-airfoil configuration is investigated using the validated methodologies. Quantitative comparisons are made with the experimental data and the results yield good agreement in terms of both near-field aerodynamics and far-field acoustic signals. The interaction between the airfoil and the vortex shedding wake is evidenced to be a major contributor to the acoustics. Further research with more detailed consideration on the turbulence modeling will be conducted in our future work.

\section{Declaration of competing interest}

The authors declare that they have no known competing financial interests or personal relationships that could have appeared to influence the work reported in this paper.

\section{Acknowledgements}

This research is supported by the 111 project on "Aircraft Complex Flows and the Control" of China (No. B17037), China Scholarship Council (No. 201706290086) and National Natural Science Foundation of China (No. 91952203). We also thank the reviewers' valuable comments, which have substantially improved the quality of our manuscript.

\section{References}

[1] T. Colonius, S.K. Lele, Computational aeroacoustics: progress on nonlinear problems of sound generation, Prog. Aerosp. Sci. 40 (2004) 345-416, https://doi.org/10.1016/j. paerosci.2004.09.001.

[2] A. Filippone, Aircraft noise prediction, Prog. Aerosp. Sci. 68 (2014) 27-63, https://doi.org/10.1016/j.paerosci.2014.02.001.

[3] M. Wang, J.B. Freund, S.K. Lele, Computational prediction of flow-generated sound, Annu. Rev. Fluid Mech. 38 (2006) 483-512, https://doi.org/10.1146/annurev.fluid.38. 050304.092036

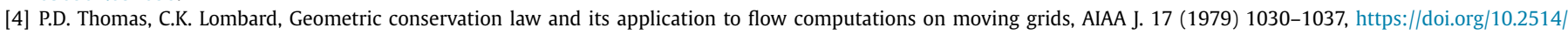
3.61273.

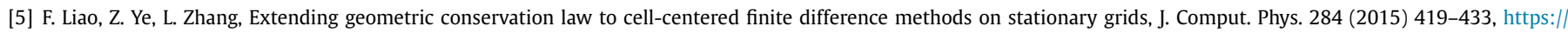
doi.org/10.1016/j.jcp.2014.12.040.

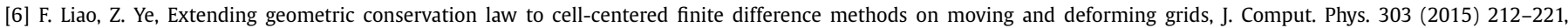
https://doi.org/10.1016/j.jcp.2015.09.032.

[7] S.K. Lele, Compact finite difference schemes with spectral-like resolution, J. Comput. Phys. 103 (1) (1992) 16-42, https://doi.org/10.1016/0021-9991(92)90324-R.

[8] C.K.W. Tam, J.C. Webb, Dispersion-relation-preserving finite difference schemes for computational acoustics, J. Comput. Phys. 107 (1993) 262-281, https://doi.org/10. $1006 /$ jcph.1993.1142.

[9] J.W. Kim, Optimised boundary compact finite difference schemes for computational aeroacoustics, J. Comput. Phys. 225 (2007) 995-1019, https://doi.org/10.1016/j.jcp. 2007.01.008.

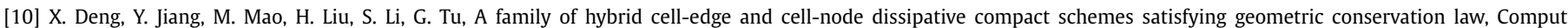
Fluids 116 (2015) 29-45, https://doi.org/10.1016/j.compfluid.2015.04.015.

[11] P.R. Spalart, Detached-Eddy simulation, Annu. Rev. Fluid Mech. 41 (2009) 181-202, https://doi.org/10.1146/annurev.fluid.010908.165130. 


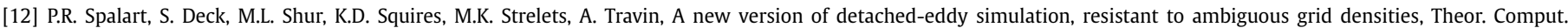
Fluid Dyn. 20 (2006) 181-195, https://doi.org/10.1007/s00162-006-0015-0.

[13] N. Chauvet, S. Deck, L. Jacquin, Zonal detached Eddy simulation of a controlled propulsive jet, AIAA J. 45 (2007) 2458-2473, https://doi.org/10.2514/1.28562.

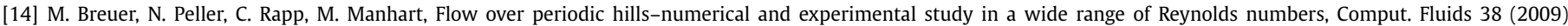
433-457, https://doi.org/10.1016/j.compfluid.2008.05.002.

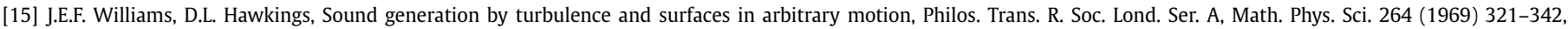
https://doi.org/10.1098/rsta.1969.0031.

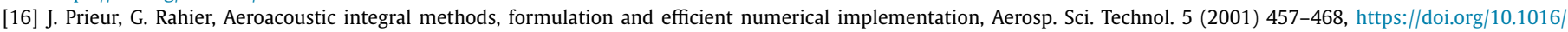
S1270-9638(01)01123-3.

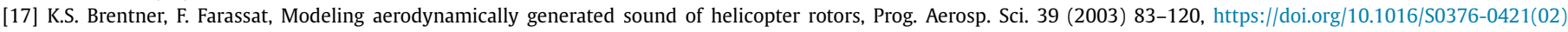
00068-4.

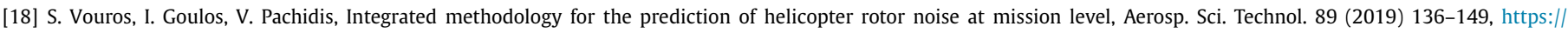
doi.org/10.1016/j.ast.2019.03.061.

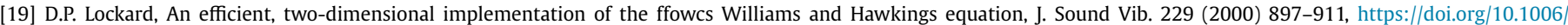
jsvi.1999.2522.

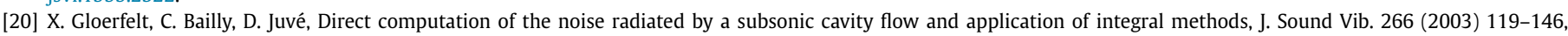
https://doi.org/10.1016/S0022-460X(02)01531-6.

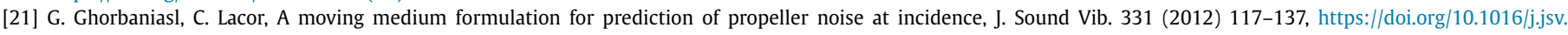
2011.08.018.

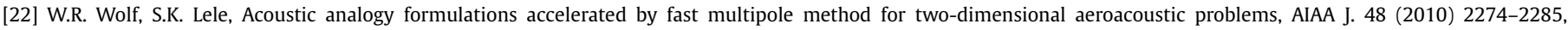
https://doi.org/10.2514/1.J050338.

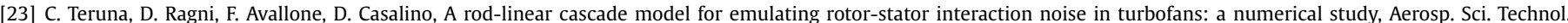
90 (2019) 275-288, https://doi.org/10.1016/j.ast.2019.04.047.

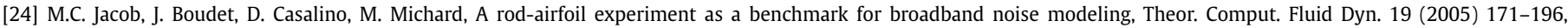
https://doi.org/10.1007/s00162-004-0108-6.

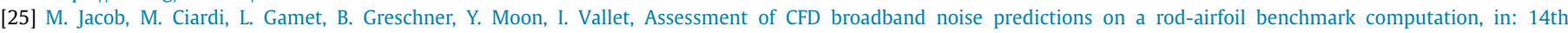
AIAA/CEAS Aeroacoustics Conf. (29th AIAA Aeroacoustics Conf.), American Institute of Aeronautics and Astronautics, Reston, Virginia, 2008.

[26] J. Blazek, Computational Fluid Dynamics: Principles and Applications, Elsevier, 2015.

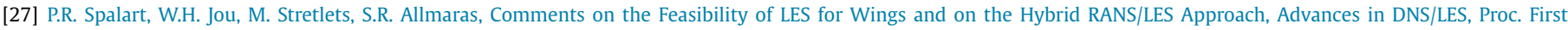
AFOSR Int. Conf. DNS/LES, 1997, pp. 137-147.

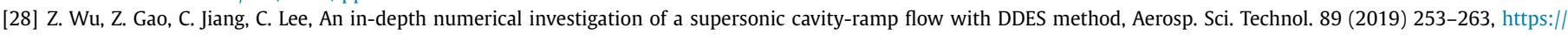
doi.org/10.1016/j.ast.2019.03.055.

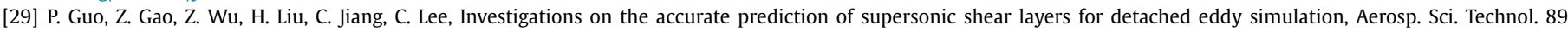
(2019) 46-57, https://doi.org/10.1016/j.ast.2019.03.045.

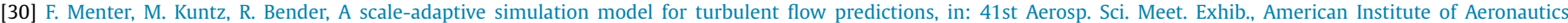
and Astronautics, Reston, Virginia, 2003.

[31] D. Casalino, An advanced time approach for acoustic analogy predictions, J. Sound Vib. 261 (2003) 583-612, https://doi.org/10.1016/S0022-460X(02)00986-0.

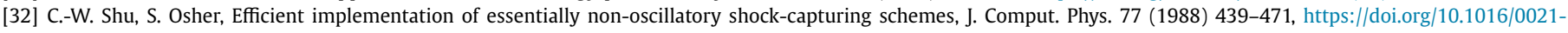
9991(88)90177-5.

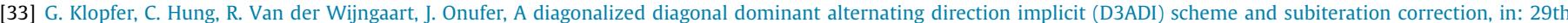
AIAA, Fluid Dyn. Conf., American Institute of Aeronautics and Astronautics, Reston, Virginia, 1998.

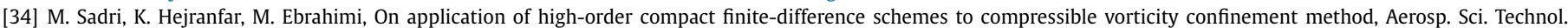
46 (2015) 398-411, https://doi.org/10.1016/j.ast.2015.07.023.

[35] F. Liao, G.W. He, High-order adapter schemes for cell-centered finite difference method, J. Comput. Phys. 403 (2020), https://doi.org/10.1016/j.jcp.2019.109090.

[36] J. Hardin, J. Ristorcelli, C. Tam, in: First ICASE/LaRC Workshop on Benchmark Problems in Computational Aeroacoustics, NASA Conf, Hampton, VA, 1995.

[37] J.W. Kim, D.J. Lee, Fourth computational aeroacoustics (CAA) workshop on benchmark problems, in: Proc. Third CAA Work. Benchmark Probl., 2000.

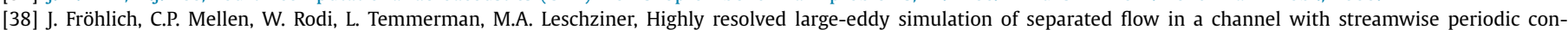
strictions, J. Fluid Mech. 526 (2005) 19-66, https://doi.org/10.1017/S0022112004002812.

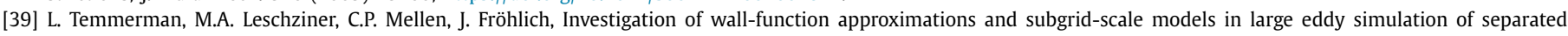
flow in a channel with streamwise periodic constrictions, Int. J. Heat Fluid Flow 24 (2003) 157-180, https://doi.org/10.1016/S0142-727X(02)00222-9.

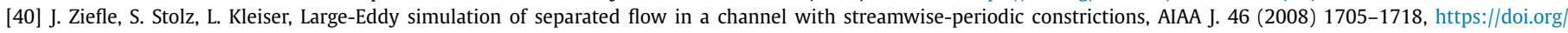
$10.2514 / 1.33891$.

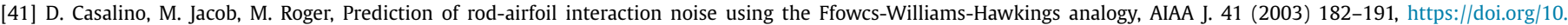
$2514 / 2.1959$.

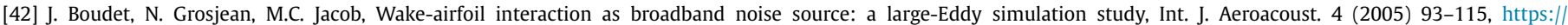
doi.org/10.1260/1475472053730093.

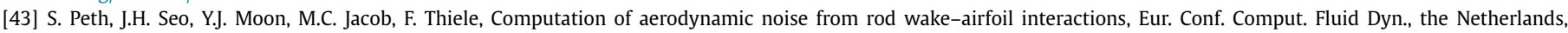
http://proceedings.fyper.com/eccomascfd2006/documents/306.pdf, 2006.

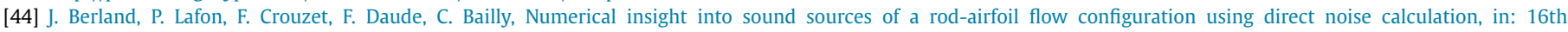
AIAA/CEAS Aeroacoustics Conf. (31st AIAA Aeroacoustics Conf.), 2010

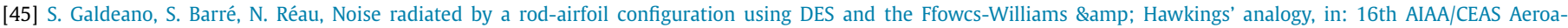
coustics Conf., American Institute of Aeronautics and Astronautics, Reston, Virginia, 2010.

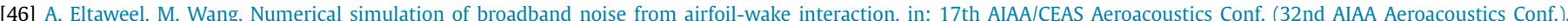
American Institute of Aeronautics and Astronautics, Reston, Virginia, 2011.

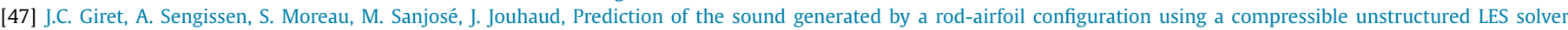
and a FW-H analogy, in: 18th AIAA/CEAS Aeroacoustics Conf. (33rd AIAA Aeroacoustics Conf.), American Institute of Aeronautics and Astronautics, Reston, Virginia, 2012.

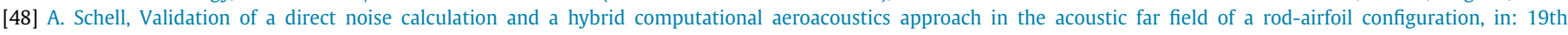
AIAA/CEAS Aeroacoustics Conf., American Institute of Aeronautics and Astronautics, Reston, Virginia, 2013.

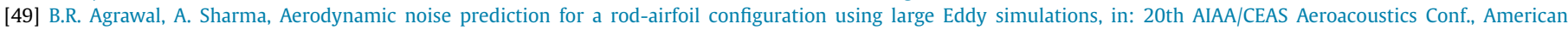
Institute of Aeronautics and Astronautics, Reston, Virginia, 2014.

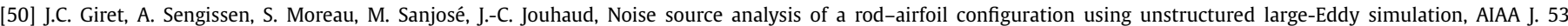
(2015) 1062-1077, https://doi.org/10.2514/1.J053371.

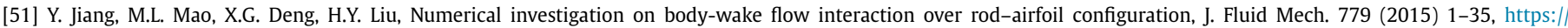
doi.org/10.1017/jfm.2015.419.

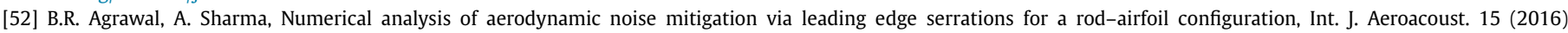
734-756, https://doi.org/10.1177/1475472X16672322.

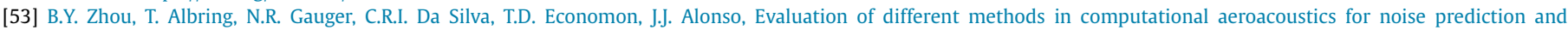
minimization of a rodairfoil configuration, in: 24th Int. Congr. Sound Vib. ICSV 2017, 2017.

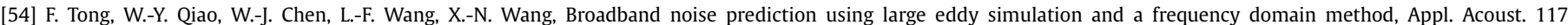
(2017) 94-105, https://doi.org/10.1016/j.apacoust.2016.11.001. 


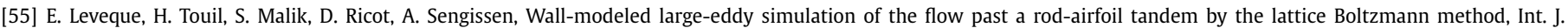
Numer. Methods Heat Fluid Flow 28 (5) (2018) 1096-1116, https://doi.org/10.1108/HFF-06-2017-0258.

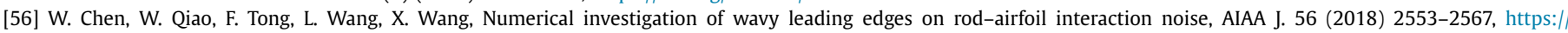
doi.org/10.2514/1.J055825.

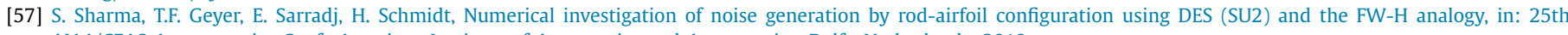
AIAA/CEAS Aeroacoustics Conf., American Institute of Aeronautics and Astronautics, Delft, Netherlands, 2019.

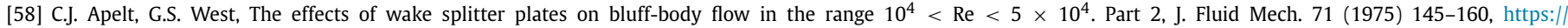
doi.org/10.1017/S0022112075002479.

[59] C. Norberg, Pressure forces on a circular cylinder in cross flow, in: Bluff-Body Wakes, Dyn. Instab., Springer Berlin Heidelberg, Berlin, Heidelberg, 1993, pp. 275-278.

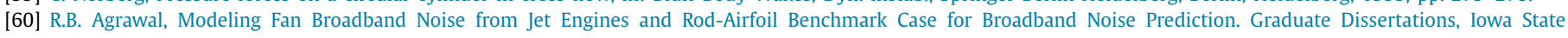
University, 2015. 